\title{
Liquid Crystalline Compounds in the Thiophene Series, Part $7^{+}$ Synthesis and Characterization of Vinylenes with Two Mesogenic Groups Each Containing Three Aromatic Systems with Different Connecting Units
}

\author{
Gerhard Koßmehl*, Frank Dirk Hoppe \\ Institut für Organische Chemie der Freien Universität Berlin, Takustraße 3, \\ D-14195 Berlin \\ Z. Naturforsch. 48b, 1807-1820 (1993); received April 9, 1993 \\ Liquid Crystalline (LC) Thiophene Compounds, Vinylenes with LC-Phases, \\ Mesogenic Groups \\ Compounds with two mesogenic groups without terminal alkyl chains or other groups con- \\ taining thiophene systems have been prepared from $\alpha . \omega$-bis(5-formyl-2-thienyl)alkanes \\ (series 1) and various phosphonium salts by Wittig-reaction. The chemical structures of the \\ new compounds have been characterized by their elemental analyses, IR and ${ }^{1} \mathrm{H}$ NMR spec- \\ tra and MS. The liquid crystalline properties of these compounds were characterized by dif- \\ ferential scanning calorimetry (DSC) and polarizing microscopy. Mesogenic cores with one \\ thiophene system and two benzene systems give rise to liquid crystalline phases. These com- \\ pounds with one vinylene group in each core (series $\mathbf{2}$ and $\mathbf{3}$ ) are mostly monotropic, those \\ with two or three vinylene groups are enantiotropic liquid crystalline. Exchanging one viny- \\ lene group by a azomethine group in each core reduces the temperature range of the meso- \\ phase or destroys the liquid crystallinity completely. In contrast to E,E-1-(5-butyl-2-thienyl- \\ vinyl)-4-(styryl)benzene (9) with no mesophase the corresponding all-E- $\alpha . \omega$-bis $\{5-[4-(4-$-sty- \\ ryl)styryl]-2-thienyl\}alkanes (series 5) show over a wide temperature range with increasing \\ inner chain length more and more high ordered mesophases. all-E- $\alpha . \omega$-Bis $\{5-[4-(4-p h e n y l-$ \\ buta-1.3-dienyl)styryl]-2-thienyl\}alkanes (series 7) decompose in their mesophases.
}

\section{Introduction}

In a previous paper we have shown that compounds with two mesogenic groups (see Scheme 1) containing thiophene systems in the aromatic unit are able to form liquid crystalline mesophases [1]. Such compounds are also named "LC-dimers" in literature. The thermal behaviour of these compounds is very different depending on the length and number of methylene groups in the inner flexible chain. The aim of this work was to compare more in depth the difference in thermal behaviour of the compounds by varying the mesogenic core containing thiophene systems. So we synthesized compounds without terminal alkyl chains or other groups (see Scheme 1) and mostly without units containing heteroatoms in the mesogenic groups (series $\mathbf{2}, \mathbf{3}, \mathbf{4}, \mathbf{5}, \mathbf{7})$ because the influence of the sulphur atom in the thiophene systems on the meso-

\footnotetext{
* Reprint requests to Prof. G. Koßmehl.

+ Part 6: G. Koßmehl, F. D. Hoppe, B. Hirsch, Z. Naturforsch. $48 \mathrm{~b}, 826$ (1993).

Verlag der Zeitschrift für Naturforschung, D-72072 Tübingen

0932-0776/93/1200-1807/\$01.00/0
}

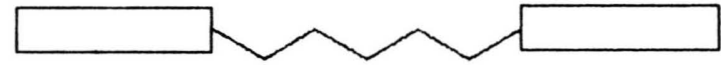

Scheme 1. General schematic formula of a compound with two mesogenic groups ("LC-dimer") and without terminal alkyl chains or other groups.

phases should be therefore dominant (see Scheme 2). For better comparison of the thermal behaviour of compounds with one and two mesogenic groups of the same structure we synthesized and characterized also the compound 9 (see Scheme 3).

\section{Synthesis}

The synthesis of the new compounds starts from the $\alpha . \omega$-bis(5-formyl-2-thienyl)alkanes (1) and some different phosphonium salts which are known in the literature (see Scheme 2) $[2-4,11]$. The dialdehydes $\mathbf{1 a - 1 1}$ are described in a previous paper [1].

The Wittig-reaction was carried out by two different methods. The first started with lithium methanolate in absol. methanol following a synthesis of Bellinger et al. [5] (method A) and the second one with potassium carbonate and dibenzo[18]crown-6 following a description of M. Boden [6] (method B). 


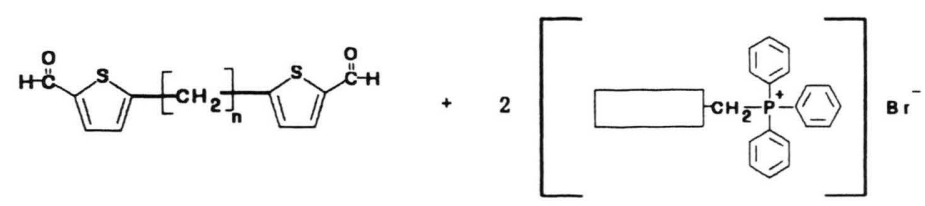

Method A

ethanol or methanol

+ lithium methanolate (1M)
Method B

$\mathrm{K}_{2} \mathrm{CO}_{3} /$ dibenzo[18]crown-6

xylene or dioxane reflux
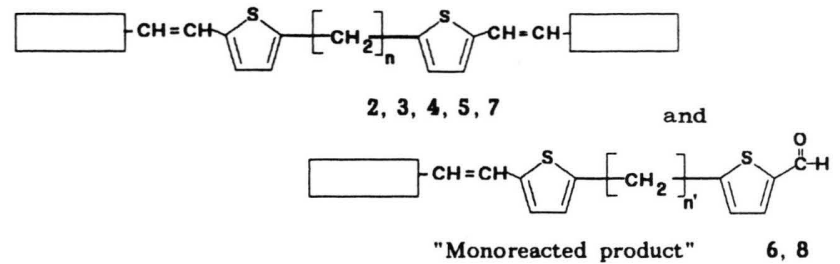

\begin{tabular}{|lccc}
\hline Compound & $n$ & $\begin{array}{c}\text { Monoreacted } \\
\text { product }\end{array}$ & $\begin{array}{c}\text { Method of } \\
\text { preparation }\end{array}$
\end{tabular}

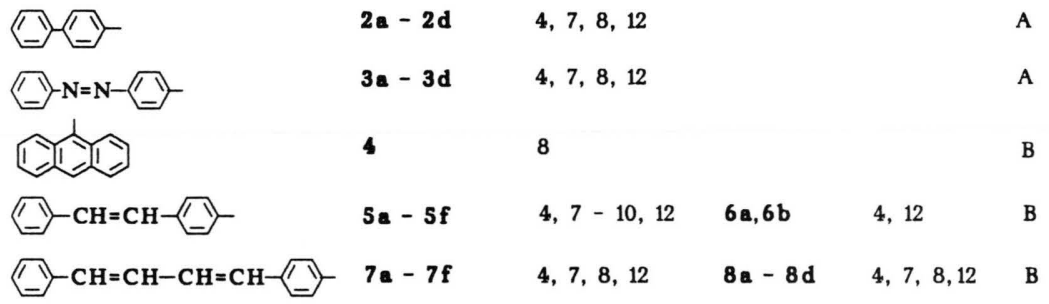

Scheme 2. Synthesis of the compounds $2-8$.
The second method was a phase-transfer-catalyst reaction and, in contrast to the first method, need no protic solvent. The phase-transfer-catalyst reaction in aprotic solvents is necessary for the synthesis of the compound 4 and series 5,7 and 9 because the corresponding phosphonium salt reacted in the presence of protic solvents and a base dominant to the methyl derivatives as shown in Scheme 4. A similar side-reaction was shown for benzyltriphenylphosphorane which reacted to toluene $[7,8]$ and for 2-thienylmethyltriphenylphosphorane which changes to 2-methylthiophene [9]. The consequence was that the yield of the desired compounds

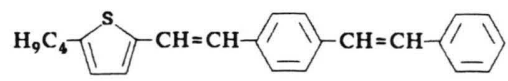

Scheme 3. Formula of 9. decreased dramatically. So for $\mathbf{4}$ respectively we could get the product only by method B. It seems the difference between the stability of the phosphonium salts can be explained by different stabilization mechanism of the intermediate carbanion which is shown in Scheme 4. Phosphonium salts with vinylene groups or the anthracenyl group could stabilize the carbanion more effectively by mesomerization than the other phosphonium salts. Moreover for method B we have varied the solvent and base for different chain lengths of the series 5 (see Table I). Only in xylene or dioxane with the base $\mathrm{K}_{2} \mathrm{CO}_{3}$ the yield is good but in comparison to the yields for related compounds published by $\mathrm{M}$. Boden [6] (about 90\%) not completely satisfactory. This difference in the yields could be explained by the different solubility of the synthesized compounds. For the synthesis of all compounds the re- 


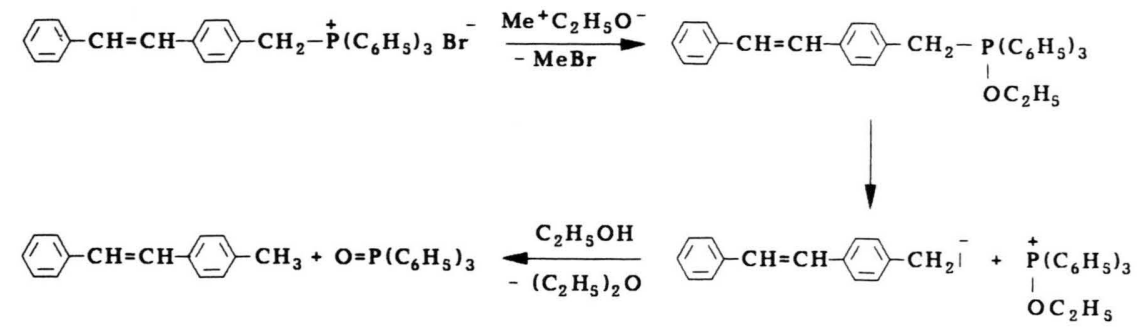

Scheme 4. The decomposition of the 4-styrylbenzylphosphonium salt in presence of an alcoholate [10]. action was therefore not complete. Therefore also the product was isolated where only one aldehyde group of the dialdehyde $\mathbf{1}$ had reacted with a phosphonium salt. In the following we call it "monoreacted product" (see Scheme 2 ). But only in a few cases the "monoreacted product" $(6 \mathbf{a}, \mathbf{6} \mathbf{b}, \mathbf{8 a - 8 d})$ can be really purified. The vinylenes (excluding the monoreacted products) were transformed into the all-E-isomers by refluxing the product $8 \mathrm{~h}$ in absol. toluene with a catalytic amount of iodine. The pure all-E-isomers of the monoreacted products are only available got by recrystallization from various solvents.

The chemical structures of all new compounds have been characterized by their elemental analyses, their IR and ${ }^{1} \mathrm{H}$ NMR spectra and MS. The electronic spectra confirm the chromophoric systems.

Table I. Preparation of the E,E-1.8-bis[5-(9-anthracenylvinyl)-2-thienyl]octane (4) and the series all-E$\alpha . \omega$-bis[5-(4-styrylstyryl)-2-thienyl]alkanes $(5 \mathbf{a}-\mathbf{5 f})$ with different bases and solvents.

\begin{tabular}{lrllr}
$\begin{array}{l}\text { Com- } \\
\text { pound }\end{array}$ & $n$ & $\begin{array}{l}\text { Solvent } \\
\text { (absol.) }\end{array}$ & Base & $\begin{array}{l}\text { Yield } \\
(\%)\end{array}$ \\
\hline $\mathbf{4}$ & 8 & xylene & $\mathrm{K}_{2} \mathrm{CO}_{3}$ & 38.2 \\
$\mathbf{5 a}$ & 4 & dioxane & $\mathrm{K}_{2} \mathrm{CO}_{3}$ & 38.0 \\
$\mathbf{5 b}$ & 7 & xylene & $\mathrm{K}_{2} \mathrm{CO}_{3}$ & 65.0 \\
$\mathbf{5 c}$ & 8 & tetrahydrofurane & $\mathrm{CH}_{3} \mathrm{COOK}$ & 9.3 \\
$\mathbf{5 d}$ & 9 & dioxane & $\mathrm{K}_{2} \mathrm{CO}_{3}$ & 69.4 \\
$\mathbf{5 d}$ & 9 & xylene & $\mathrm{K}_{2} \mathrm{CO}_{3}$ & 69.2 \\
$\mathbf{5 e}$ & 10 & tetrahydrofurane & $\mathrm{K}_{2} \mathrm{CO}_{3}$ & 35.6 \\
$\mathbf{5 f}$ & 12 & dioxane & $\mathrm{KF}^{2}$ & 19.2 \\
\hline
\end{tabular}

\section{Experimental}

IR spectra were measured with a Perkin-Elmer580-B spectrometer. ${ }^{1} \mathrm{H}$ NMR spectra were measured at $270 \mathrm{MHz}$ using a Bruker WH 270 instrument, the chemical shifts are given relative to tetramethylsilane (TMS). Mass spectra were measured with a Varian MAT-112-S instrument. Elemental analyses were performed with a Perkin-ElmerRecorder 56. The phase transitions were determined by Heraeus TA 500 instrument with a scanning speed of $10^{\circ} \mathrm{C} / \mathrm{min}$ and sometimes $20^{\circ} \mathrm{C} / \mathrm{min}$ for heating and cooling runs. The first and second heating cycle was used for the determination of the phase transition temperatures and enthalpies. Optical investigations were carried out with a Zeiss polarization microscope using a Lincam heating regulation system.

\section{General synthesis for}

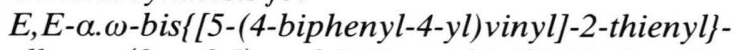

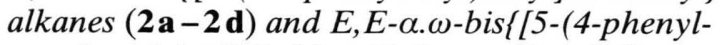
azophenylvinyl)-2-thienyl]alkanes (3a-3 d)

(method A; see Scheme 2)

The reaction of the $\alpha . \omega$-bis(5-formyl-2-thienyl)alkanes (1a-1f) [1] and phosphonium salts to the vinylene derivatives $2 \mathbf{a - 2 d}$ and $3 \mathbf{a - 3 d}$ was carried out in absol. ethanol or absol. methanol under an argon atmosphere respectively for $\mathbf{2 a - 2 d}$ with triphenyl(4-phenylbenzyl)phosphonium bromide [2] and for 3a-3d triphenyl(4-phenylazobenzyl)phosphonium bromide [11]. The reaction was started with different volumes of $1 \mathrm{M} \mathrm{CH}_{3} \mathrm{OLi}$ in absol. methanol where the intermediate ylene coloured the solution with luminosity colours. The reaction solution was stirred for $3 \mathrm{~h}$. The end of the reaction was detected by TLC in $\mathrm{CHCl}_{3}$ where the product is in the front $\left(R_{f}\right.$ around 1$)$, the "monoproduct" more in the "middle" ( $R_{f}$ around 0.5$)$ and the educt near at the starting point of the chromatogram. For further transformation more base (in $1 \mathrm{ml}$ portions) was given to the solution. For the compounds $2 \mathbf{a}-2 \mathrm{~d}$ and $3 \mathbf{a}-\mathbf{3} \mathrm{d}$ most of the product was precipitated. For purification see below General description for the purification of all compounds, prepared by method A and B.

The isolation of the direacted and monoreacted product was done by liquid chromatography on neutral silicagel (Woelm) where the mobile phase was first pure hexane and lateron a mixture of chloroform and hexane. The first fraction is the E/Zmixture of the pure direacted product, the second fraction is mostly pure E/Z-mixture of the mono- 
reacted product. Both derivatives were refluxed $8 \mathrm{~h}$ in $50 \mathrm{ml}$ absol. toluene with a catalytic amount of iodine for changing the E/Z-mixture to the all-Eproduct. Respectively for derivatives with long inner chain the second fraction was not the pure "monoreacted product" so a second liquid-chromatographic run as before was done. For further purification the products have been crystallized from various solvents and dried in vacuo over $\mathrm{P}_{2} \mathrm{O}_{5}$.

\section{E,E-1.4-Bis[5-(4-biphenylvinyl)-2-thienyl]butane} (2a)

From $0.400 \mathrm{~g}(1.44 \mathrm{mmol}) \mathbf{1 a}($ with $n=4), 1.600 \mathrm{~g}$ (3 mmol) triphenyl-(4-phenylbenzyl)phosphonium bromide [2] in $50 \mathrm{ml}$ absol. ethanol and $8 \mathrm{ml}$ lithium methanolate (1 $\mathrm{M}$ in methanol), orange-yellow crystals from toluene; m.p. $244^{\circ} \mathrm{C}$; yield $0.438 \mathrm{~g}$ $(53 \%)$.

$$
\begin{array}{ccc}
\mathrm{C}_{40} \mathrm{H}_{34} \mathrm{~S}_{2}(578.8) & \\
\text { Calcd } & \text { C } 83.00 & \text { H } 5.92 \%, \\
\text { Found } & \text { C } 82.97 & \text { H } 5.88 \% .
\end{array}
$$

IR $(\mathrm{KBr}), \quad v\left(\mathrm{~cm}^{-1}\right)=3060(\mathrm{w}), \quad 3035(\mathrm{w})$ $v(\mathrm{Ar}-\mathrm{C}-\mathrm{H}) ; \quad 2918(\mathrm{~m}), \quad 2848(\mathrm{~m}) \quad v(\mathrm{C}-\mathrm{H})$; $1625(\mathrm{~m}) \quad v\left(\mathrm{C}=\mathrm{C}_{\text {conj }}\right) ; \quad 1580(\mathrm{w}), \quad 1600(\mathrm{~s})$ $v(\mathrm{Ar}-\mathrm{C}=\mathrm{C}) ; \quad 955(\mathrm{~s}) \quad \delta(\mathrm{C}-\mathrm{H})_{\mathrm{oop}} ; \quad 822(\mathrm{~s})$ $\delta(\mathrm{Ar}-\mathrm{C}-\mathrm{H})_{\text {oop }}$.

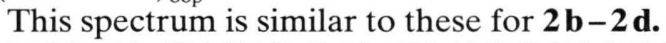

MS $(\mathrm{m} / z): 578(100)\left(\mathbf{M}^{+}\right) ; 289(11)\left(\mathbf{M}^{2+}\right) 275(62)$ $\left(\mathrm{C}_{19} \mathrm{H}_{15} \mathrm{~S}\right)^{+} ; 241(20)\left(\mathrm{C}_{19} \mathrm{H}_{13}\right)^{+} ; 165(7)\left(\mathrm{C}_{13} \mathrm{H}_{9}\right)^{+}$.

$\mathrm{UV}\left(\mathrm{CHCl}_{3}\right) \lambda(\mathrm{nm}) / \log \varepsilon: 265$ (3.53), 335 (sh), 350 (4.39), 370 (sh).

These absorptions are also typical for $\mathbf{2 b - 2 d}$.

\section{E,E-1.7-Bis[5-(4-biphenylvinyl)-2-thienyl]heptane} (2b)

From $0.460 \mathrm{~g}(1.44 \mathrm{mmol}) \mathbf{1 b}$ (with $n=7), 1.600 \mathrm{~g}$ (3 mmol) triphenyl-(4-phenylbenzyl)phosphonium bromide in $50 \mathrm{ml}$ absol. ethanol and $8 \mathrm{ml}$ lithium methanolate ( $1 \mathrm{M}$ in methanol), orange-yellow crystals from toluene; m.p. $203^{\circ} \mathrm{C}$; yield $0.643 \mathrm{~g}$ (72\%).

$$
\begin{aligned}
& \mathrm{C}_{43} \mathrm{H}_{40} \mathrm{~S}_{2} \text { (620.9) } \\
& \text { Calcd C } 83.18 \text { H 6.95\%, } \\
& \text { Found C } 83.16 \text { H 6.91\%. }
\end{aligned}
$$

MS $(m / z): 620(100)\left(\mathrm{M}^{+}\right) ; 310(13)\left(\mathrm{M}^{2+}\right) ; 275(68)$ $\left(\mathrm{C}_{19} \mathrm{H}_{15} \mathrm{~S}\right)^{+} ; 241(17)\left(\mathrm{C}_{19} \mathrm{H}_{13}\right)^{+} ; 165(6)\left(\mathrm{C}_{13} \mathrm{H}_{9}\right)^{+}$.

\section{E,E-1.8-Bis[5-(4-biphenylvinyl)-2-thienyl]octane} (2c)

From $0.480 \mathrm{~g}(1.44 \mathrm{mmol}) 1 \mathrm{c}$ (with $n=8), 1.600 \mathrm{~g}$ (3 mmol) triphenyl-(4-phenylbenzyl)phosphonium bromide in $50 \mathrm{ml}$ absol. ethanol and $8 \mathrm{ml}$ lithium methanolate (1 $\mathrm{M}$ in methanol), orange-yellow crystals from toluene; m.p. $213^{\circ} \mathrm{C}$; yield $0.593 \mathrm{~g}$ $(65 \%)$.

$$
\begin{array}{ccc}
\mathrm{C}_{44} \mathrm{H}_{42} \mathrm{~S}_{2}(634.9) & \\
\text { Calcd } & \mathrm{C} 83.23 & \mathrm{H} 6.67 \%, \\
\text { Found } & \text { C } 83.21 & \text { H } 6.64 \% .
\end{array}
$$

MS $(m / z): 634(100)\left(\mathbf{M}^{+}\right) ; 317(10)\left(\mathbf{M}^{2+}\right) ; 275(54)$ $\left(\mathrm{C}_{19} \mathrm{H}_{15} \mathrm{~S}\right)^{+} ; 241(16)\left(\mathrm{C}_{19} \mathrm{H}_{13}\right)^{+} ; 167(6)\left(\mathrm{C}_{13} \mathrm{H}_{11}\right)^{+}$.

\section{E,E-1.12-Bis[5-(4-biphenylvinyl)-2-thienyl]dodecane (2d)}

From $0.560 \mathrm{~g}(1.44 \mathrm{mmol}) \mathbf{1 f}$ (with $n=12), 1.600 \mathrm{~g}$ (3 mmol) triphenyl-(4-phenylbenzyl)phosphonium bromide in $50 \mathrm{ml}$ absol. ethanol and $8 \mathrm{ml}$ lithium methanolate (1 M in methanol), orange-yellow crystals from toluene; m.p. $199^{\circ} \mathrm{C}$; yield $0.507 \mathrm{~g}$ $(51 \%)$.

$$
\begin{aligned}
& \mathrm{C}_{48} \mathrm{H}_{50} \mathrm{~S}_{2} \text { (691.0) } \\
& \text { Calcd C } 83.67 \text { H 7.02\%, } \\
& \text { Found C } 83.16 \text { H 6.91\%. } \\
& \text { MS ( } \mathrm{m} / z): 690(100)\left(\mathbf{M}^{+}\right) ; 345(15)\left(\mathbf{M}^{2+}\right) ; 275(49) \\
& \left(\mathrm{C}_{19} \mathrm{H}_{15} \mathrm{~S}\right)^{+} ; 241(10)\left(\mathrm{C}_{19} \mathrm{H}_{13}\right)^{+} ; 167(6)\left(\mathrm{C}_{13} \mathrm{H}_{11}\right)^{+} \text {. }
\end{aligned}
$$

\section{E,E-1.4-Bis[5-(4-phenylazophenylvinyl)-2-thienyl]- butane (3 a)}

From $0.258 \mathrm{~g}(0.93 \mathrm{mmol}) \mathbf{1 a}$ (with $n=4), 1.100 \mathrm{~g}$ ( $2 \mathrm{mmol}$ ) triphenyl-(4-phenylazobenzyl)phosphonium bromide [15] in $50 \mathrm{ml}$ absol. ethanol and $8 \mathrm{ml}$ lithium methanolate (1 $\mathrm{M}$ in methanol), orange-red crystals from $\mathrm{CH}_{2} \mathrm{Cl}_{2}$ and toluene; m. p. $231^{\circ} \mathrm{C}$, cl. p. (dec.) $251^{\circ} \mathrm{C}$; yield $0.375 \mathrm{~g}(64 \%)$.

\section{$\mathrm{C}_{40} \mathrm{H}_{34} \mathrm{~N}_{4} \mathrm{~S}_{2}$ (634.9) \\ Calcd C 75.68 H 5.40 N 8.82\%, \\ Found C 75.67 H 5.38 N 8.81\%.}

IR $(\mathrm{KBr}), \quad v\left(\mathrm{~cm}^{-1}\right)=3070(\mathrm{w}), 3040(\mathrm{w})$ $v(\mathrm{Ar}-\mathrm{C}-\mathrm{H}) ; 3020(\mathrm{w}) v\left(\mathrm{C}=\mathrm{C}_{\text {trans }}-\mathrm{H}\right) ; 2930(\mathrm{w})$, $2860(\mathrm{w}) v(\mathrm{C}-\mathrm{H}) ; 1620(\mathrm{~m}) v\left(\mathrm{C}=\mathrm{C}_{\text {conj. }}\right) ; 1470(\mathrm{~s})$, 1460 (s), $1440(\mathrm{~s}) v\left(\right.$ aromat); $950(\mathrm{~s}) \delta(\mathrm{C}-\mathrm{H})_{\text {oop }}$.

These absorptions are also typical for $\mathbf{3 b - 3} \mathbf{b}$.

MS (m/z): $634(8)\left(\mathrm{M}^{+}\right) ; 545(20)\left(\mathrm{C}_{34} \mathrm{H}_{31} \mathrm{~N}_{3} \mathrm{~S}_{2}\right)^{+}$; $456(5)\left(\mathrm{C}_{28} \mathrm{H}_{28} \mathrm{~N}_{2} \mathrm{~S}_{2}\right)^{+} ; 303(6)\left(\mathrm{C}_{19} \mathrm{H}_{15} \mathrm{~N}_{2} \mathrm{~S}\right)^{+} ; 214(36)$ $\left.\mathrm{C}_{13} \mathrm{H}_{12} \mathrm{NS}\right)^{+}$.

UV (KBr) $\lambda(\mathrm{nm}): 245, \underline{365}, 463$.

This spectrum is similar to these for $\mathbf{3 b} \mathbf{b} \mathbf{3} \mathbf{d}$.

\section{E,E-1.7-Bis[5-(4-phenylazophenylvinyl)-2-thienyl]- heptane (3b)}

From $0.297 \mathrm{~g}(0.93 \mathrm{mmol}) \mathbf{1 b}$ (with $n=7), 1,100 \mathrm{~g}$ ( $2 \mathrm{mmol}$ ) triphenyl-(4-phenylazobenzyl)phospho- 
nium bromide in $50 \mathrm{ml}$ absol. ethanol and $10 \mathrm{ml} \mathrm{li-}$ thium methanolate (1 $\mathrm{M}$ in methanol), orange-red crystals from $\mathrm{CH}_{2} \mathrm{Cl}_{2}$ and toluene; m. p. $196^{\circ} \mathrm{C}$; yield $0.484 \mathrm{~g}(77 \%)$.

$$
\begin{array}{rrrr}
\mathrm{C}_{43} \mathrm{H}_{40} \mathrm{~N}_{4} \mathrm{~S}_{2}(676.9) & & \\
\text { Calcd } & \mathrm{C} 76.29 & \mathrm{H} 5.95 & \mathrm{~N} 8.28 \%, \\
\text { Found } & \text { C 76.25 } & \text { H } 5.93 & \text { N 8.28\%. }
\end{array}
$$

MS $(\mathrm{m} / \mathrm{z}): 676(14)\left(\mathrm{M}^{+}\right) ; 587(20)\left(\mathrm{C}_{37} \mathrm{H}_{37} \mathrm{~N}_{3} \mathrm{~S}_{2}\right)^{+}$; $214(28)\left(\mathrm{C}_{13} \mathrm{H}_{12} \mathrm{NS}\right)^{+}$.

\section{E,E-1.8-Bis[5-(4-phenylazophenylvinyl)-2-thienyl]- octane (3 c)}

From $0.310 \mathrm{~g}(0.93 \mathrm{mmol}) \mathbf{1 c}($ with $n=8), 1.100 \mathrm{~g}$ ( $2 \mathrm{mmol}$ ) triphenyl-(4-phenylazobenzyl)phosphonium bromide in $50 \mathrm{ml}$ absol. ethanol and $10 \mathrm{ml} \mathrm{li-}$ thium methanolate (1 $\mathrm{M}$ in methanol), orange-red crystals from $\mathrm{CH}_{2} \mathrm{Cl}_{2}$ and toluene; m. p. $215^{\circ} \mathrm{C}$; yield $0.203 \mathrm{~g}(62 \%)$.

$$
\begin{array}{cccc}
\mathrm{C}_{44} \mathrm{H}_{42} \mathrm{~N}_{4} \mathrm{~S}_{2}(691.0) & & \\
\text { Calcd } & \mathrm{C} 76.48 & \mathrm{H} 6.13 & \mathrm{~N} 8.11 \%, \\
\text { Found } & \mathrm{C} 76.44 & \mathrm{H} 6.10 & \text { N } 8.10 \% .
\end{array}
$$

MS $(\mathrm{m} / z): 690(3)\left(\mathrm{M}^{+}\right) ; 602(38)\left(\mathrm{C}_{38} \mathrm{H}_{40} \mathrm{~N}_{3} \mathrm{~S}_{2}\right)^{+}$; $303(2)\left(\mathrm{C}_{19} \mathrm{H}_{15} \mathrm{~N}_{2} \mathrm{~S}\right)^{+} ; 214(2)\left(\mathrm{C}_{13} \mathrm{H}_{12} \mathrm{NS}\right)^{+}$.

\section{E,E-1.12-Bis[5-(4-phenylazophenylvinyl)-2-thienyl]- dodecane $(\mathbf{3} \mathbf{d})$}

From $0.365 \mathrm{~g}(0.93 \mathrm{mmol}) \mathbf{1 f}$ (with $n=12), 1.100 \mathrm{~g}$ ( $2 \mathrm{mmol}$ ) triphenyl-(4-phenylazobenzyl)phosphonium bromide in $50 \mathrm{ml}$ absol. ethanol and $10 \mathrm{ml} \mathrm{li-}$ thium methanolate (1 M in methanol), orange-red crystals from $\mathrm{CH}_{2} \mathrm{Cl}_{2}$ and toluene; m. p. $179^{\circ} \mathrm{C}$; yield $0.562 \mathrm{~g}(81 \%)$.

$$
\begin{array}{cccc}
\mathrm{C}_{48} \mathrm{H}_{50} \mathrm{~N}_{4} \mathrm{~S}_{2}(747.1) & & \\
\text { Calcd } & \mathrm{C} 77.17 & \mathrm{H} 6.75 & \mathrm{~N} 7.50 \%, \\
\text { Found } & \mathrm{C} 77.00 & \mathrm{H} 6.65 & \text { N 7.48\%. }
\end{array}
$$

MS (m/z): $746(13)\left(\mathrm{M}^{+}\right) ; 658(66)\left(\mathrm{C}_{42} \mathrm{H}_{48} \mathrm{~N}_{3} \mathrm{~S}_{2}\right)^{+}$; 568 (47) $\left(\mathrm{C}_{36} \mathrm{H}_{44} \mathrm{~N}_{2} \mathrm{~S}_{2}\right)^{+} ; 303$ (9) $\left(\mathrm{C}_{19} \mathrm{H}_{15} \mathrm{~N}_{2} \mathrm{~S}\right)^{+} ; 214$ (97) $\left(\mathrm{C}_{13} \mathrm{H}_{12} \mathrm{NS}\right)^{+}$.

General synthesis of the compounds $4,5 a-5 f$, 7a-7d and 9 (method B).

The phase-transfer-catalyst (PTK) reaction was done by a procedure given by R. M. Boden [6] where the base is $\mathrm{K}_{2} \mathrm{CO}_{3}$ and the PTK dibenzo[18] crown-6. The compounds were synthesized mostly in $30 \mathrm{ml}$ xylene under an argon atmosphere with $\alpha . \omega$-bis(5-formyl-2-thienyl)alkanes (1a-1f) [1], phosphonium salt, $\mathrm{K}_{2} \mathrm{CO}_{3}$ and dibenzo[18] crown-6 where the phosphonium salt and the base were only suspended. While heating the reaction solution about $18 \mathrm{~h}$ most of the product was precipitated. For removing $\mathrm{K}_{2} \mathrm{CO}_{3}$ the unpurified product was washed repeatedly with water. This crude product was dried over $\mathrm{P}_{2} \mathrm{O}_{5}$ by vacuum and after this transformed by refluxing $8 \mathrm{~h}$ in absol. toluene with a catalytic amount of iodine from the E/Z-isomer to the all-E product. The product was recrystallized from different solvents. The product which was still soluble in the reaction solution is to be purified by liquid chromatography (see above).

\section{E,E-1.8-Bis[5-(9-anthracenylvinyl)-2-thienyl]- octane (4)}

From $0.330 \mathrm{~g}(1 \mathrm{mmol}) 1 \mathrm{c}$ (with $n=8), 1.000 \mathrm{~g}$ ( $2 \mathrm{mmol})$ triphenyl-(9-anthracenylmethyl)phosphonium chloride, $2.800 \mathrm{~g}(20 \mathrm{mmol}) \mathrm{K}_{2} \mathrm{CO}_{3}$ and $0.007 \mathrm{~g}$ (0.02 mmol) dibenzo[18]crown-6 in $30 \mathrm{ml}$ absol. xylene, $18 \mathrm{~h}$ reflux; light orange powder from $\mathrm{EtOH} / \mathrm{THF}$; m.p. $110^{\circ} \mathrm{C}$; yield $0.252 \mathrm{~g}(37 \%)$.

$$
\begin{aligned}
& \mathrm{C}_{48} \mathrm{H}_{42} \mathrm{~S}_{2} \text { (683.0) } \\
& \text { Calcd C } 84.41 \text { H 6.20\%, } \\
& \text { Found C } 84.37 \text { H } 6.21 \% \text {. }
\end{aligned}
$$

IR $(\mathrm{KBr}), v\left(\mathrm{~cm}^{-1}\right)=3045(\mathrm{~m}) v\left(\mathrm{C}-\mathrm{H}_{\mathrm{Ar}}\right) ; 3013(\mathrm{w})$ $v\left(\mathrm{C}=\mathrm{C}-\mathrm{H}_{\text {trans }}\right) ; 2925(\mathrm{~s}), 2851(\mathrm{~m}) v_{\text {sy }}\left(\mathrm{CH}_{2}\right) ; 1620$ (m) $v\left(\mathrm{C}=\mathrm{C}_{\text {aromatic conj. }}\right) ; 1471(\mathrm{w}), 1441(\mathrm{~m}) \delta_{\text {as }}\left(\mathrm{CH}_{2}\right)$ or thiophene; 1213 (vw) thiophene; $956(\mathrm{~m})$ $\omega\left(\mathrm{C}=\mathrm{C}-\mathrm{H}_{\text {trans }}\right) ; 799(\mathrm{~m}) 2.5$-thienylene.

${ }^{1} \mathrm{H}$ NMR $\left(\mathrm{CDCl}_{3}\right) \delta(\mathrm{ppm})=6.78-8.39$ (multiplett, $26 \mathrm{H}$, aromatic protons); $2.89(\mathrm{~m}, 4 \mathrm{H}$, $\left.\mathrm{Th}-\mathrm{CH}_{2}\right) ; 1.78\left(\mathrm{~m}, 4 \mathrm{H}, \mathrm{Th}-\mathrm{CH}_{2}-\mathrm{CH}_{2}\right) ; 1.46(\mathrm{~m}$, $\left.8 \mathrm{H}, \mathrm{Th}-\left(\mathrm{CH}_{2}\right)_{2}-\left(\mathrm{CH}_{2}\right)_{2}\right)$.

MS ( $\mathrm{m} / \mathrm{z}): 682(91)\left(\mathbf{M}^{+}\right) ; 341(12)\left(\mathbf{M}^{2+}\right) ; 299(26)$ $\left(\mathrm{C}_{21} \mathrm{H}_{15} \mathrm{~S}\right)^{+} ; 285(22)\left({ }_{20} \mathrm{H}_{13} \mathrm{~S}\right)^{+} ; 265(62)\left(\mathrm{C}_{21} \mathrm{H}_{13}\right)^{+} ; 252$ (14) $\left(\mathrm{C}_{20} \mathrm{H}_{12}\right)^{+}$.

\section{all-E-1.4-Bis[5-(4-styrylstyryl)-2-thienyl]butane (5a)}

From $0.278 \mathrm{~g}(1 \mathrm{mmol}) \mathbf{1 a}$ (with $n=4), 1.07 \mathrm{~g}$ (2 mmol) triphenyl-(4-styrylbenzyl)phosphonium bromide [13] in $30 \mathrm{ml}$ absol. dioxane, $2.800 \mathrm{~g}$ $(20 \mathrm{mmol}) \mathrm{K}_{2} \mathrm{CO}_{3}$ and $0.007 \mathrm{~g}(0.02 \mathrm{mmol})$ dibenzo[18]crown- $6,18 \mathrm{~h}$ reflux; orange crystals from toluene; m.p. $288^{\circ} \mathrm{C}$, cl.p. (dec.) $316^{\circ} \mathrm{C}$; yield $0.239 \mathrm{~g}$ $(38 \%)$.

$$
\begin{array}{crc}
\mathrm{C}_{44} \mathrm{H}_{38} \mathrm{~S}_{2}(630.9) & \\
\text { Calcd } & \mathrm{C} 83.76 & \mathrm{H} 6.07 \%, \\
\text { Found } & \mathrm{C} 83.70 & \mathrm{H} 6.02 \% .
\end{array}
$$

$\mathrm{IR}(\mathrm{KBr}), v\left(\mathrm{~cm}^{-1}\right)=3060(\mathrm{~m}) v(\mathrm{Ar}-\mathrm{C}-\mathrm{H}) ; 3022$ (m) $v\left(\mathrm{C}=\mathrm{C}_{\text {trans }}-\mathrm{H}\right) ; 2920(\mathrm{~m}), 2850(\mathrm{~m}) v(\mathrm{C}-\mathrm{H})$; $1625(\mathrm{~m}) v\left(\mathrm{C}=\mathrm{C}_{\text {trans }}\right) ; 1595(\mathrm{~m}) v(\mathrm{Ph}-\mathrm{C}=\mathrm{C}) ; 1510$ (m) $v(\mathrm{Ar}-\mathrm{C}=\mathrm{C}) ; 960(\mathrm{vs}) \delta\left(\mathrm{C}=\mathrm{C}_{\text {trans }}-\mathrm{H}\right)_{\text {oop }} ; 815$ (vs) $\delta(\mathrm{Th}-\mathrm{C}-\mathrm{H})_{\text {oop }}$.

These absorptions are also typical for $\mathbf{5 b - 5} \mathbf{f}$.

MS ( $\mathrm{m} / \mathrm{z})$ : $630(100)\left(\mathbf{M}^{+}\right) ; 315(10)\left(\mathbf{M}^{2+}\right) ; 301(19)$ $\left(\mathrm{C}_{21} \mathrm{H}_{17} \mathrm{~S}\right)^{+} ; 267$ (2) $\left(\mathrm{C}_{21} \mathrm{H}_{15}\right)^{+}$.

$\mathrm{UV}(\mathrm{KBr}) \lambda(\mathrm{nm}): 248,328(\mathrm{sh}), \underline{341}, 382$ (sh), 413.

This spectrum is similar to these for $\mathbf{5 b - 5 f}$. 


\section{all-E-1.7-Bis[5-(4-styrylstyryl)-2-thienyl]heptane}

(5b)

From $0.320 \mathrm{~g}(1 \mathrm{mmol}) \mathbf{1 b}$ (with $n=7), 1.07 \mathrm{~g}$ (2 mmol) triphenyl-(4-styrylbenzyl)phosphonium bromide in $30 \mathrm{ml}$ absol. xylene, $2.800 \mathrm{~g}(20 \mathrm{mmol})$ $\mathrm{K}_{2} \mathrm{CO}_{3}$ and $0.007 \mathrm{~g}(0.02 \mathrm{mmol})$ dibenzo[18]crown-6, $18 \mathrm{~h}$ reflux; light yellow crystals from toluene; m.p. $246{ }^{\circ} \mathrm{C}$, cl. p. $262{ }^{\circ} \mathrm{C}$; yield $0.437 \mathrm{~g}(65 \%)$.

The "monoreacted product" $\mathbf{6}$ a was also isolated by liquid chromatography.

$\mathrm{C}_{47} \mathrm{H}_{44} \mathrm{~S}_{2}(673.0)$

Calcd C 83.88 H $6.59 \%$,

Found C 83.75 H $6.53 \%$.

MS $(m / z): 672(100)\left(\mathbf{M}^{+}\right) ; 336(15)\left(\mathrm{M}^{2+}\right) ; 315(2)$ $\left(\mathrm{C}_{22} \mathrm{H}_{19} \mathrm{~S}\right)^{+} ; 301(66)\left(\mathrm{C}_{21} \mathrm{H}_{17} \mathrm{~S}\right)^{+} ; 267(9)\left(\mathrm{C}_{21} \mathrm{H}_{15}\right)^{+}$.

all-E-1.8-Bis[5-(4-styrylstyryl)-2-thienyl]octane (5 c)

From $0.334 \mathrm{~g}(1 \mathrm{mmol}) \mathbf{1}$ (with $n=8), 1.070 \mathrm{~g}$ ( $2 \mathrm{mmol}$ ) triphenyl-(4-styrylbenzyl)phosphonium bromide in $30 \mathrm{ml}$ absol. THF, $3.900 \mathrm{~g} \mathrm{(40} \mathrm{mmol)} \mathrm{po-}$ tassium acetat and $0.007 \mathrm{~g}(0.02 \mathrm{mmol})$ dibenzo[18]crown-6, $18 \mathrm{~h}$ reflux; light yellow crystals from toluene; m.p. $256^{\circ} \mathrm{C}$, cl.p. $273^{\circ} \mathrm{C}$; yield $0.064 \mathrm{~g}$ $(9.3 \%)$.

$$
\begin{array}{ccc}
\mathrm{C}_{48} \mathrm{H}_{46} \mathrm{~S}_{2}(687.0) & \\
\text { Calcd } & \mathrm{C} 83.92 & \text { H } 6.75 \%, \\
\text { Found } & \text { C } 83.89 & \text { H } 6.72 \% .
\end{array}
$$

MS $(\mathrm{m} / z): 686(100)\left(\mathrm{M}^{+}\right) ; 343(16)\left(\mathrm{M}^{2+}\right) ; 315(2)$ $\left(\mathrm{C}_{22} \mathrm{H}_{19} \mathrm{~S}\right)^{+} ; 301(64)\left(\mathrm{C}_{21} \mathrm{H}_{17} \mathrm{~S}\right)^{+} ; 267(10)\left(\mathrm{C}_{21} \mathrm{H}_{15}\right)^{+}$.

\section{all-E-1.9-Bis[5-(4-styrylstyryl)-2-thienyl]nonane}

(5d)

From $0.348 \mathrm{~g}(1 \mathrm{mmol}) \mathbf{1 d}$ (with $n=9), 1.070 \mathrm{~g}$ ( $2 \mathrm{mmol}$ ) triphenyl-(4-styrylbenzyl)phosphonium bromide in $30 \mathrm{ml}$ absol. dioxane or xylene, $2.800 \mathrm{~g}$ $(20 \mathrm{mmol}) \mathrm{K}_{2} \mathrm{CO}_{3}$ and $0.007 \mathrm{~g}(0.02 \mathrm{mmol})$ dibenzo[18]crown-6, $18 \mathrm{~h}$ reflux; light yellow crystals from toluene; m.p. $227^{\circ} \mathrm{C}$, cl.p. $259^{\circ} \mathrm{C}$; yield $0.486 \mathrm{~g}$ $(69.4 \%)$ or $0.484 \mathrm{~g}(69.4 \%$ in dioxane, $69.2 \%$ in xylene).

$$
\begin{aligned}
& \mathrm{C}_{49} \mathrm{H}_{48} \mathrm{~S}_{2} \text { (701.1) } \\
& \text { Calcd C } 83.95 \text { H } 6.90 \% \text {, } \\
& \text { Found C } 83.93 \text { H 6.91\%. }
\end{aligned}
$$

MS $(\mathrm{m} / \mathrm{z}): 700(100)\left(\mathbf{M}^{+}\right) ; 350(15)\left(\mathbf{M}^{2+}\right) ; 314(1)$ $\left(\mathrm{C}_{22} \mathrm{H}_{18} \mathrm{~S}\right)^{+} ; 301(32)\left(\mathrm{C}_{21} \mathrm{H}_{17} \mathrm{~S}\right)^{+} ; 267(4)\left(\mathrm{C}_{21} \mathrm{H}_{15}\right)^{+}$.

\section{all-E-1.10-Bis[5-(4-styrylstyryl)-2-thienyl]decane} (5e)

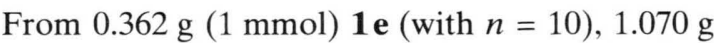
(2 mmol) triphenyl-(4-styrylbenzyl)phosphonium bromide in $30 \mathrm{ml}$ absol. THF, $2.800 \mathrm{~g}(20 \mathrm{mmol})$
$\mathrm{K}_{2} \mathrm{CO}_{3}$ and $0.007 \mathrm{~g}(0.02 \mathrm{mmol})$ dibenzo[18]crown-6, $18 \mathrm{~h}$ reflux; light yellow crystals from toluene; m.p. $246^{\circ} \mathrm{C}$, cl.p. $257^{\circ} \mathrm{C}$; yield $0.254 \mathrm{~g}(35.6 \%)$.

$$
\begin{array}{ccc}
\mathrm{C}_{50} \mathrm{H}_{50} \mathrm{~S}_{2}(715.1) & \\
\text { Calcd } & \mathrm{C} 83.98 & \mathrm{H} 7.05 \%, \\
\text { Found } & \mathrm{C} 83.95 & \mathrm{H} 7.02 \% .
\end{array}
$$

MS ( $m / z): 714(100)\left(\mathbf{M}^{+}\right) ; 357(13)\left(\mathbf{M}^{2+}\right) ; 314(1)$ $\left(\mathrm{C}_{22} \mathrm{H}_{18} \mathrm{~S}\right)^{+} ; 301(42)\left(\mathrm{C}_{21} \mathrm{H}_{17} \mathrm{~S}\right)^{+} ; 267(3)\left(\mathrm{C}_{21} \mathrm{H}_{15}\right)^{+}$.

all-E-1.12-Bis[5-(4-styrylstyryl)-2-thienyl]dodecane (5f)

From $0.390 \mathrm{~g}(1 \mathrm{mmol}) \mathbf{1 f}$ (with $n=12), 1.070 \mathrm{~g}$ ( $2 \mathrm{mmol}$ ) triphenyl-(4-styrylbenzyl)phosphonium bromide in $30 \mathrm{ml}$ absol. dioxane, $2.320 \mathrm{~g}$ (40 mmol) $\mathrm{KF}$ and $0.007 \mathrm{~g}(0.02 \mathrm{mmol})$ dibenzo[18]crown-6, $18 \mathrm{~h}$ reflux; light yellow crystals from toluene; m.p. $238^{\circ} \mathrm{C}$, cl. p. $247^{\circ} \mathrm{C}$; yield $0.142 \mathrm{~g}(19.2 \%)$.

The "monoreacted product" $\mathbf{6} \mathbf{b}$ was also isolated by liquid chromatography.

$$
\begin{array}{ccc}
\mathrm{C}_{52} \mathrm{H}_{54} \mathrm{~S}_{2}(743.1) & \\
\text { Calcd } & \mathrm{C} 84.05 & \mathrm{H} 7.32 \%, \\
\text { Found } & \mathrm{C} 84.03 & \mathrm{H} 7.30 \% .
\end{array}
$$

MS $(\mathrm{m} / \mathrm{z}): 742(100)\left(\mathrm{M}^{+}\right) ; 371(6)\left(\mathrm{M}^{2+}\right) ; 314(3)$ $\left(\mathrm{C}_{22} \mathrm{H}_{18} \mathrm{~S}\right)^{+} ; 301(38)\left(\mathrm{C}_{21} \mathrm{H}_{17} \mathrm{~S}\right)^{+} ; 267(5)\left(\mathrm{C}_{21} \mathrm{H}_{15}\right)^{+}$.

all-E-1-(5-Formyl-2-thienyl)-4-[5-(4-styrylstyryl)2-thienyl]butane (6a)

From the synthesis of $\mathbf{5 a}$ (see above). The "monoreacted product" was isolated by liquid chromatography [silica-gel/first $\mathrm{CHCl}_{3}$-hexane $(1: 5)$, later $\mathrm{CHCl}_{3}$-hexane $\left.(1: 1)\right]$ of the crude product where the first fraction is $\mathbf{5} \mathbf{a}$ and the second fraction is 6a, light yellow powder from $\mathrm{THF} / \mathrm{EtOH}$; m.p. $184{ }^{\circ} \mathrm{C}$; yield $0.016 \mathrm{~g}(3.5 \%)$.

$$
\begin{array}{rrr}
\mathrm{C}_{29} \mathrm{H}_{26} \mathrm{~S} & \mathrm{O}(454.6) \\
\text { Calcd } & \mathrm{C} 76.61 & \mathrm{H} 5.76 \%, \\
\text { Found } & \mathrm{C} 76.55 & \mathrm{H} 5.70 \% .
\end{array}
$$

$\mathrm{IR}(\mathrm{KBr}), v\left(\mathrm{~cm}^{-1}\right)=3080(\mathrm{~m}) v(\mathrm{Ar}-\mathrm{C}-\mathrm{H}) ; 3022$ (m) $v\left(\mathrm{C}=\mathrm{C}_{\text {trans }}-\mathrm{H}\right) ; 2920(\mathrm{~m}), 2850(\mathrm{~m}) v(\mathrm{C}-\mathrm{H})$; $1660(\mathrm{~s}) v(\mathrm{C}=\mathrm{O}) ; 1595(\mathrm{~m}) v(\mathrm{Ph}-\mathrm{C}=\mathrm{C}) ; 1515(\mathrm{w})$ $v(\mathrm{Ar}-\mathrm{C}=\mathrm{C}) ; 955(\mathrm{~s}) \quad \delta\left(\mathrm{C}=\mathrm{C}_{\text {trans }}-\mathrm{H}\right)_{\mathrm{oop}} ; 810$ (s) $\delta(\mathrm{Th}-\mathrm{C}-\mathrm{H})_{\text {oop }}$.

This spectrum is similar to this for $\mathbf{6} \mathbf{b}$.

${ }^{1} \mathrm{H} \operatorname{NMR}\left(\mathrm{CDCl}_{3}\right) \delta(\mathrm{ppm})=9.82(\mathrm{~s}, 1 \mathrm{H}$, $-\mathrm{CH}=\mathrm{O}) ; 7.6\left(\mathrm{~d}, 1 \mathrm{H}, J_{4-3}=3.5 \mathrm{~Hz}, \mathrm{Th}-\mathrm{H}_{4}\right)$; $7.07-7.56\left(\mathrm{~m}, 12 \mathrm{H}, \mathrm{Bz}_{9}, \operatorname{VinH}_{1} \mathrm{H}_{1^{\prime}} \mathrm{H}_{2^{\prime}}\right) ; 6.89(\mathrm{~d}$, $\left.1 \mathrm{H}, J_{3-4}=3.5 \mathrm{~Hz}, \mathrm{Th}-\mathrm{H}_{3}\right) ; 6.86\left(\mathrm{~d}, 1 \mathrm{H}, J_{4^{\prime}-3^{\prime}}=3.5 \mathrm{~Hz}\right.$, $\left.\mathrm{Th}-\mathrm{H}_{4^{\prime}}\right) ; 6.79\left(\mathrm{~d}, 1 \mathrm{H}, J_{2-1}=16 \mathrm{~Hz}, \mathrm{VinH}_{2}\right) ; 6.66(\mathrm{~d}$, $\left.1 \mathrm{H}, J_{3^{\prime} \mathrm{A}^{\prime}}=3.5 \mathrm{~Hz}, \mathrm{Th}-\mathrm{H}_{3^{\prime}}\right) ; 2.93(\mathrm{t}, 2 \mathrm{H}, J=8 \mathrm{~Hz}$, $\left.\underline{\mathrm{H}}_{2} \mathrm{C}-\mathrm{Th}-\mathrm{CHO}\right) ; 2.86(\mathrm{t}, 2 \mathrm{H}, \quad J=8 \mathrm{~Hz}$, $\left.\underline{\mathrm{H}}_{2} \mathrm{C}-\mathrm{Th}-\mathrm{CH}=\mathrm{CH}\right) ; 1.8\left(\mathrm{~m}, 4 \mathrm{H}, \mathrm{Th}-\mathrm{CH}_{2}-\mathrm{C}_{2}\right)$. 
MS $(\mathrm{m} / z): 454(100)\left(\mathrm{M}^{+}\right) ; 227(3)\left(\mathrm{M}^{2+}\right) ; 328(3)$ $\left(\mathrm{C}_{23} \mathrm{H}_{21} \mathrm{~S}\right)^{+} ; 314(3)\left(\mathrm{C}_{22} \mathrm{H}_{19} \mathrm{~S}\right)^{+} ; 301(63)\left(\mathrm{C}_{21} \mathrm{H}_{17} \mathrm{~S}\right)^{+}$; $267(12)\left(\mathrm{C}_{21} \mathrm{H}_{15}\right)^{+}$.

UV $\left(\mathrm{CHCl}_{3}\right) \lambda(\mathrm{nm}) / \log \varepsilon: 250$ (3.46), 298 (3.69), 358 (sh), 373 (4.24), 395 (sh).

These absorptions are also typical for $\mathbf{6 b}$.

all-E-1-(5-Formyl-2-thienyl)-12-[5-(4-styrylstyryl)2-thienyl]dodecane (6b)

From the synthesis of $\mathbf{5} \mathbf{f}$ (see above). The "monoreacted product" was isolated by liquid chromatography [silica-gel/first $\mathrm{CHCl}_{3}$-hexane (1:5), later $\mathrm{CHCl}_{3}$-hexane $\left.(1: 1)\right]$ of the crude product where the first fraction is $\mathbf{5} \mathbf{f}$ and the second fraction is $\mathbf{6} \mathbf{b}$, light yellow powder from THF/EtOH; m. p. $157^{\circ} \mathrm{C}$; yield $0.048 \mathrm{~g}(8.4 \%)$.

$$
\begin{array}{rrr}
\mathrm{C}_{37} \mathrm{H}_{42} \mathrm{~S}_{2} \mathrm{O}(566.9) & \\
\text { Calcd } & \text { C 78.40 } & \mathrm{H} 8.00 \%, \\
\text { Found } & \text { C 78.31 } & \text { H 7.94\%. }
\end{array}
$$

${ }^{1} \mathrm{H}$ NMR $\left(\mathrm{CDCl}_{3}\right) \delta(\mathrm{ppm})=9.82(\mathrm{~s}, 1 \mathrm{H}$, $-\mathrm{CH}=\mathrm{O}) ; 7.6\left(\mathrm{~d}, 1 \mathrm{H}, J_{4-3}=3.5 \mathrm{~Hz}, \mathrm{Th}-\mathrm{H}_{4}\right)$; $7.07-7.56\left(\mathrm{~m}, 12 \mathrm{H}, \mathrm{Bz}_{9}, \mathrm{VinH}_{1} \mathrm{H}_{1}, \mathrm{H}_{2^{\prime}}\right) ; 6.89$ (d, $\left.1 \mathrm{H}, J_{3-4}=3.5 \mathrm{~Hz}, \mathrm{Th}-\mathrm{H}_{3}\right) ; 6.86\left(\mathrm{~d}, 1 \mathrm{H}, J_{4^{\prime}-3^{\prime}}=3.5 \mathrm{~Hz}\right.$, $\left.\mathrm{Th}-\mathrm{H}_{4^{\prime}}\right) ; 6.79\left(\mathrm{~d}, 1 \mathrm{H}, J_{2-1}=16 \mathrm{~Hz}, \mathrm{VinH}_{2}\right) ; 6.66(\mathrm{~d}$, $\left.1 \mathrm{H}, J_{3^{\prime}-4^{\prime}}=3.5 \mathrm{~Hz}, \mathrm{Th}-\mathrm{H}_{3^{\prime}}\right) ; 2.86(\mathrm{t}, 2 \mathrm{H}, J=8 \mathrm{~Hz}$, $\left.\underline{\mathrm{H}}_{2} \mathrm{C}-\mathrm{Th}-\mathrm{CHO}\right) ; 2.78(\mathrm{t}, 2 \mathrm{H}, \quad J=8 \mathrm{~Hz}$, $\left.\underline{\mathrm{H}}_{2} \mathrm{C}-\mathrm{Th}-\mathrm{CH}=\mathrm{CH}\right) ; 1.7\left(\mathrm{~m}, 4 \mathrm{H}, \mathrm{Th}-\mathrm{CH}_{2}-\mathrm{CH}_{2}\right)$; $1.32\left(\mathrm{~m}, 16 \mathrm{H}, \mathrm{Th}-\left(\mathrm{CH}_{2}\right)_{2}-\left(\mathrm{CH}_{2}\right)_{4}\right)$.

MS $(\mathrm{m} / \mathrm{z}): 566(100)\left(\mathrm{M}^{+}\right) ; 283(5)\left(\mathrm{M}^{2+}\right) ; 328$ (1) $\left(\mathrm{C}_{23} \mathrm{H}_{21} \mathrm{~S}\right)^{+} ; 314$ (4) $\left(\mathrm{C}_{22} \mathrm{H}_{19} \mathrm{~S}\right)^{+} ; 301$ (49) $\left(\mathrm{C}_{21} \mathrm{H}_{17} \mathrm{~S}\right)^{+}$; 267 (9) $\left(\mathrm{C}_{21} \mathrm{H}_{15}\right)^{+}$.

all-E-1.4-Bis\{5-[4-(4-phenyl-buta-1.3-dienyl)styryl]2-thienyl) (7 a)

From $0.278 \mathrm{~g}(1 \mathrm{mmol}) \mathbf{1} \mathbf{a}$ (with $n=4), 1.120 \mathrm{~g}$ (2 mmol) triphenyl-[4-(4-phenyl-1.3-butadienyl)benzyl]phosphonium bromide [4] in $30 \mathrm{ml}$ absol. xylene, $2.800 \mathrm{~g}(20 \mathrm{mmol}) \mathrm{K}_{2} \mathrm{CO}_{3}$ and $0.007 \mathrm{~g}$ (0.02 mmol) dibenzo[18]crown-6, $18 \mathrm{~h}$ reflux; gold coloured yellow crystals from xylene; m.p. $286^{\circ} \mathrm{C}$, cl.p. (dec.) $293^{\circ} \mathrm{C}$; yield $0.150 \mathrm{~g}(22 \%)$. The "monoreacted product" 8 a was also isolated by liquid chromatography (see below).

$\mathrm{C}_{48} \mathrm{H}_{42} \mathrm{~S}_{2}(683.0)$

Calcd C 84.41 H 6.20\%,

Found C 84.38 H 6.22\%.

IR (KBr), $\nu\left(\mathrm{cm}^{-1}\right)=3090(\mathrm{w}), 3058(\mathrm{~m})$ $v(\mathrm{Ar}-\mathrm{C}-\mathrm{H}) ; 3020(\mathrm{~m}) v\left(\mathrm{C}=\mathrm{C}_{\text {trans }}-\mathrm{H}\right) ; 2920(\mathrm{~s})$, $2850(\mathrm{~m}) v(\mathrm{C}-\mathrm{H}) ; 1595(\mathrm{w}) v(\mathrm{Ph}-\mathrm{C}=\mathrm{C}) ; 1510(\mathrm{~m})$ $v(\mathrm{Ar}-\mathrm{C}=\mathrm{C}) ; 1495(\mathrm{~m}), 1465(\mathrm{~m}), 1445$ (m) $v(\mathrm{Ar})$; $990(\mathrm{vs}) \delta\left(\mathrm{C}=\mathrm{C}_{\text {trans }}-\mathrm{H}\right)_{\mathrm{oop}} ; 805(\mathrm{~m}) \delta(\mathrm{Th}-\mathrm{C}-\mathrm{H})_{\text {oop }}$.
These absorptions are also typical for $\mathbf{7 b - 7} \mathbf{d}$.

MS ( $\mathrm{m} / z)$ : $682(100)\left(\mathrm{M}^{+}\right) ; 341(10)\left(\mathrm{M}^{2+}\right) ; 327$ (46) $\left(\mathrm{C}_{23} \mathrm{H}_{19} \mathrm{~S}\right)^{+} ; 452$ (1) $\left(\mathrm{C}_{30} \mathrm{H}_{28} \mathrm{~S}_{2}\right)^{+}$.

UV (KBr) $\lambda(\mathrm{nm}): 252,327$ (sh), $\underline{360}, 375(\mathrm{sh}), 400$ (sh), 430.

all-E-1.7-Bis\{5-[4-(4-phenyl-buta-1.3-dienyl)styryl]2-thienyllheptane (7 b)

From $0.320 \mathrm{~g}(1 \mathrm{mmol}) \mathbf{1} \mathbf{b}$ (with $n=7), 1.120 \mathrm{~g}$ (2 mmol) triphenyl-[4-(4-phenyl-1.3-butadienyl)benzyl]phosphonium bromide in $30 \mathrm{ml}$ absol. dioxane, $2.800 \mathrm{~g}$ (20 mmol) $\mathrm{K}_{2} \mathrm{CO}_{3}$ and $0.007 \mathrm{~g}$ (0.02 mmol) dibenzo[18]crown-6, $18 \mathrm{~h}$ reflux; yellow crystals from xylene; m. p. $253^{\circ} \mathrm{C}$, cl. p. (dec.) $273^{\circ} \mathrm{C}$; yield $0.210 \mathrm{~g}(29 \%)$.

The "monoreacted product" $\mathbf{8} \mathbf{b}$ was also isolated by liquid chromatography.

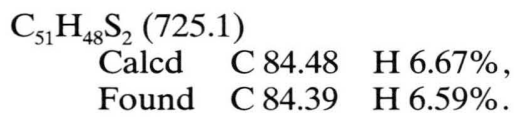

MS ( $\mathrm{m} / \mathrm{z}): 724$ (100) $\left(\mathrm{M}^{+}\right) ; 362$ (12) $\left(\mathrm{M}^{2+}\right) ; 494$ (1) $\left(\mathrm{C}_{33} \mathrm{H}_{34} \mathrm{~S}_{2}\right)^{+} ; 327(35)\left(\mathrm{C}_{23} \mathrm{H}_{19} \mathrm{~S}\right)^{+}$.

all-E-1.8-Bis\{5-[4-(4-phenyl-buta-1.3-dienyl)styryl]2-thienylloctane (7 c)

From $0.334 \mathrm{~g}$ (1 mmol) $\mathbf{1 c}$ (with $n=8), 1.120 \mathrm{~g}$ (2 mmol) triphenyl-[4-(4-phenyl-1.3-butadienyl)benzyl]phosphonium bromide in $30 \mathrm{ml}$ absol. dioxane, $2.800 \mathrm{~g}(20 \mathrm{mmol}) \mathrm{K}_{2} \mathrm{CO}_{3}$ and $0.007 \mathrm{~g}$ (0.02 mmol) dibenzo[18]crown-6, $18 \mathrm{~h}$ reflux; yellow crystals from xylene; m. p. $259^{\circ} \mathrm{C}$, cl.p. (dec.) $278^{\circ} \mathrm{C}$; yield $0.236 \mathrm{~g}(32 \%)$.

The "monoreacted product" $8 \mathbf{c}$ was also isolated by liquid chromatography.

$\mathrm{C}_{52} \mathrm{H}_{50} \mathrm{~S}_{2}(739.1)$

Calcd C 84.51 H 6.82\%,

Found C 84.50 H 6.79\%.

$\operatorname{MS}(\mathrm{m} / z): 738(100)\left(\mathrm{M}^{+}\right) ; 369$ (12) $\left(\mathrm{M}^{2+}\right) ; 508$ (1) $\left(\mathrm{C}_{34} \mathrm{H}_{36} \mathrm{~S}_{2}\right)^{+} ; 327(42)\left(\mathrm{C}_{23} \mathrm{H}_{19} \mathrm{~S}\right)^{+}$.

all-E-1.12-Bis\{5-[4-(4-phenyl-buta-1.3-dienyl)styryl]-2-thienyl]dodecane (7 d)

From $0.390 \mathrm{~g}$ (1 mmol) $\mathbf{1 f}$ (with $n=12), 1.120 \mathrm{~g}$ (2 mmol) triphenyl-[4-(4-phenyl-1.3-butadienyl)benzyl]phosphonium bromide in $30 \mathrm{ml}$ absol. xylene, $2.800 \mathrm{~g} \mathrm{(20} \mathrm{mmol}) \mathrm{K}_{2} \mathrm{CO}_{3}$ and $0.007 \mathrm{~g}(0.02 \mathrm{mmol}) \mathrm{di}-$ benzo[18]crown-6, $18 \mathrm{~h}$ reflux; yellow brown crystals from xylene; m.p. $249^{\circ} \mathrm{C}$, cl.p. (dec.) $257^{\circ} \mathrm{C}$; yield $0.191 \mathrm{~g}(24 \%)$.

The "monoreacted product" $8 \mathbf{d}$ was also isolated by liquid chromatography. 
$\mathrm{C}_{56} \mathrm{H}_{58} \mathrm{~S}_{2}$ (795.2)

Calcd C 84.59 H 7.35\%,

Found C 84.53 H 7.40\%.

MS ( $m / z): 794(100)\left(\mathbf{M}^{+}\right) ; 397(9)\left(\mathbf{M}^{2+}\right) ; 564$ (94)

$\left(\mathrm{C}_{38} \mathrm{H}_{44} \mathrm{~S}_{2}\right)^{+} ; 327(36)\left(\mathrm{C}_{23} \mathrm{H}_{19} \mathrm{~S}\right)^{+}$.

all-E-1-(5-Formyl-2-thienyl)-4-\{5-[4-(4-phenyl-buta1.3-dienyl)styryl]-2-thienyl\}butane $(\mathbf{8} \mathbf{a})$

From the synthesis of $\mathbf{7}$ a (see above). The "monoreacted product" was isolated by liquid chromatography [silica-gel/first $\mathrm{CHCl}_{3}$-hexane $(1: 5)$, later $\mathrm{CHCl}_{3}$-hexane (1:1)] of the crude product where the first fraction is $\mathbf{7 a}$ and the second fraction is $8 \mathbf{a}$, light yellow powder from THF/EtOH; m. p. $197^{\circ} \mathrm{C}$, cl.p. $213{ }^{\circ} \mathrm{C}$; yield $0.101 \mathrm{~g}(21 \%)$.

$$
\begin{array}{rr}
\mathrm{C}_{31} \mathrm{H}_{28} \mathrm{~S} \\
\text { Calcd } \mathrm{O}(480.7) \\
\text { Found } 77.46 & \mathrm{H} 77.87 \% \text {, } \\
\text { F } 77.61 \% .
\end{array}
$$

$\operatorname{IR}(\mathrm{KBr}), v\left(\mathrm{~cm}^{-1}\right)=3080(\mathrm{~m}), v(\mathrm{Ar}-\mathrm{C}-\mathrm{H}) ; 3020$ (m) $v\left(\mathrm{C}=\mathrm{C}_{\text {trans }}-\mathrm{H}\right) ; 2920(\mathrm{~s}), 2850(\mathrm{~m}) v(\mathrm{C}-\mathrm{H})$; $1695(\mathrm{~s}), 1665(\mathrm{~s}) v(\mathrm{C}=\mathrm{O}) ; 1550(\mathrm{~m}) v(\mathrm{Ar}-\mathrm{C}=\mathrm{C})$; $980(\mathrm{vs}) \delta\left(\mathrm{C}=\mathrm{C}_{\text {trans }}-\mathrm{H}\right)_{\text {oop }} ; 805(\mathrm{~m}) \delta(\mathrm{Th}-\mathrm{C}-\mathrm{H})_{\text {oop }}$.

These absorptions are also typical for $\mathbf{8 b - 8 d}$.

${ }^{1} \mathrm{H}$ NMR $\left(\mathrm{CDCl}_{3}\right) \delta(\mathrm{ppm})=9.81(\mathrm{~s}, 1 \mathrm{H}$, $-\mathrm{C} \underline{\mathrm{H}}=\mathrm{O}) ; 7.63\left(\mathrm{~d}, 1 \mathrm{H}, J_{4-3}=3.7 \mathrm{~Hz}, \mathrm{Th}-\mathrm{H}_{4}\right)$; $6.53-7.00\left(\mathrm{~m}, 18 \mathrm{H}, \mathrm{Bz}_{4}, \mathrm{Vin}_{\mathrm{H}} ; \mathrm{Th}_{\mathrm{H}}\right) ; 2.96(\mathrm{t}$, $\left.2 \mathrm{H}, J=8 \mathrm{~Hz}, \underline{\mathrm{H}}_{2} \mathrm{C}-\mathrm{Th}-\mathrm{CHO}\right) ; 2.86(\mathrm{t}, 2 \mathrm{H}, J=8 \mathrm{~Hz}$, $\left.\underline{\mathrm{H}}_{2} \mathrm{C}-\mathrm{Th}-\mathrm{CH}=\mathrm{CH}\right) ; 1.81\left(\mathrm{~m}, 4 \mathrm{H}, \underline{\mathrm{H}}_{2} \mathrm{C}-\mathrm{CH}_{2}-\mathrm{Th}\right)$.

MS $(\mathrm{m} / \mathrm{z}): 480(100)\left(\mathrm{M}^{+}\right) ; 240(8)\left(\mathrm{M}^{2+}\right) ; 452(8)$ $(\mathrm{M}-\mathrm{CO})^{+} ; 327(59)\left(\mathrm{C}_{23} \mathrm{H}_{19} \mathrm{~S}\right)^{+}$.

$\mathrm{UV}\left(\mathrm{CHCl}_{3}\right) \lambda(\mathrm{nm}) / \log \varepsilon: 257$ (3.52), 303 (3.52), 375 (sh), 388 (4.3), 410 (4.04).

This spectrum is similar to these for $\mathbf{8 b - 8 d}$.

all-E-1-(5-Formyl-2-thienyl)-7-\{5-[4-(4-phenyl-buta1.3-dienyl)styryl]-2-thienyllheptane $(\mathbf{8 b})$

From the synthesis of $\mathbf{7} \mathbf{b}$ (see above). The "monoreacted product" was isolated by liquid chromatography [silica-gel/first $\mathrm{CHCl}_{3}$-hexane (1:5), later $\mathrm{CHCl}_{3}$-hexane (1:1)] of the crude product where the first fraction is $\mathbf{7 b}$ and the second fraction is $\mathbf{8 b}$, light yellow powder from THF/EtOH; m.p. $177^{\circ} \mathrm{C}$; yield $0.172 \mathrm{~g} \mathrm{(33 \% )}$.

$$
\begin{aligned}
& \mathrm{C}_{34} \mathrm{H}_{34} \mathrm{~S}_{2} \mathrm{O} \text { (522.8) } \\
& \text { Calcd C 78.12 H 6.56\%, } \\
& \text { Found C78.01 H 6.54\%. }
\end{aligned}
$$

${ }^{1} \mathrm{H}$ NMR $\left(\mathrm{CDCl}_{3}\right) \delta(\mathrm{ppm})=9.81(\mathrm{~s}, 1 \mathrm{H}$, $-\mathrm{CH}=\mathrm{O}) ; 7.63\left(\mathrm{~d}, 1 \mathrm{H}, J_{4-3}=3.7 \mathrm{~Hz}, \mathrm{Th}-\mathrm{H}_{4}\right)$; $6.53-7.00\left(\mathrm{~m}, 18 \mathrm{H}, \mathrm{Bz}_{\mathrm{H}}, \mathrm{Vin}_{\mathrm{H}} ; \mathrm{Th}_{\mathrm{H}}\right) ; 2.88(\mathrm{t}$, $\left.2 \mathrm{H}, J=8 \mathrm{~Hz}, \underline{\mathrm{H}}_{2} \mathrm{C}-\mathrm{Th}-\mathrm{CHO}\right) ; 2.79(\mathrm{t}, 2 \mathrm{H}, J=8 \mathrm{~Hz}$, $\left.\underline{\mathrm{H}}_{2} \mathrm{C}-\mathrm{Th}-\mathrm{CH}=\mathrm{CH}\right) ; 1.7\left(\mathrm{~m}, 4 \mathrm{H}, \underline{\mathrm{H}}_{2} \mathrm{C}-\mathrm{CH}_{2}-\mathrm{Th}\right)$; $1.34\left(\mathrm{~m}, 6 \mathrm{H}, \mathrm{Th}-\left(\mathrm{CH}_{2}\right)_{2}-\left(\mathrm{C}_{2}\right)_{3}\right)$.
This spectrum is also typical for $\mathbf{8 c}$.

MS ( $\mathrm{m} / \mathrm{z}): 522(100)\left(\mathrm{M}^{+}\right) ; 261(13)\left(\mathrm{M}^{2+}\right) ; 494(2)$ $(\mathrm{M}-\mathrm{CO})^{+} ; 327(35)\left(\mathrm{C}_{23} \mathrm{H}_{19} \mathrm{~S}\right)^{+}$.

all-E-1-(5-Formyl-2-thienyl)-8-\{5-[4-(4-phenyl-buta1.3-dienyl)styryl]-2-thienyl\}octane (8c)

From the synthesis of $\mathbf{7}$ c (see above). The "monoreacted product" was isolated by liquid chromatography [silica-gel/first $\mathrm{CHCl}_{3}$-hexane (1:5), later $\mathrm{CHCl}_{3}$-hexane (1:1)] of the crude product where the first fraction is $\mathbf{7} \mathbf{c}$ and the second fraction is $\mathbf{8 c}$, light yellow powder from THF/EtOH; m.p. $181^{\circ} \mathrm{C}$, cl.p. $189^{\circ} \mathrm{C}$; yield $0.139 \mathrm{~g}(26 \%)$.

$$
\begin{aligned}
& \mathrm{C}_{35} \mathrm{H}_{36} \mathrm{~S}_{2} \mathrm{O} \text { (536.8) } \\
& \text { Calcd C 78.31 H 6.76\%, } \\
& \text { Found C 78.27 H 6.59\%. }
\end{aligned}
$$

MS $(\mathrm{m} / z): 536(100)\left(\mathrm{M}^{+}\right) ; 268(9)\left(\mathrm{M}^{2+}\right) ; 508(1)$ $(\mathrm{M}-\mathrm{CO})^{+} ; 327(25)\left(\mathrm{C}_{23} \mathrm{H}_{19} \mathrm{~S}\right)^{+}$.

all-E-1-(5-Formyl-2-thienyl)-12-\{5-[4-(4-phenylbuta-1.3-dienyl)styryl]-2-thienyl\}dodecane (8d)

From the synthesis of $\mathbf{7} \mathbf{d}$ (see above). The "monoreacted product" was isolated by liquid chromatography [silica-gel/first $\mathrm{CHCl}_{3}$-hexane (1:5), later $\mathrm{CHCl}_{3}$-hexane (1:1)] of the crude product where the first fraction is $\mathbf{7} \mathbf{d}$ and the second fraction is $\mathbf{8 d}$, light yellow powder from THF/EtOH; m.p. $169^{\circ} \mathrm{C}$; yield $0.243 \mathrm{~g}(41 \%)$.

$$
\begin{array}{rrr}
\mathrm{C}_{39} \mathrm{H}_{44} \mathrm{~S} & \mathrm{O}(592.9) \\
\text { Calcd } & \mathrm{C} 79.00 & \mathrm{H} 7.48 \%, \\
\text { Found } & \mathrm{C} 78.84 & \mathrm{H} 7.44 \% .
\end{array}
$$

${ }^{1} \mathrm{H}$ NMR $\left(\mathrm{CDCl}_{3}\right) \delta(\mathrm{ppm})=9.81(\mathrm{~s}, 1 \mathrm{H}$, $-\mathrm{C} \underline{\mathrm{H}}=\mathrm{O}) ; 7.63\left(\mathrm{~d}, 1 \mathrm{H}, J_{4-3}=3.7 \mathrm{~Hz}, \mathrm{Th}-\mathrm{H}_{4}\right)$; 6.53-7.00 (m, $\left.18 \mathrm{H}, \mathrm{Bz}_{\mathrm{H}_{1}}, \operatorname{Vin}_{\mathrm{H}} ; \mathrm{Th}_{\mathrm{H}}\right) ; 2.87(\mathrm{t}$, $\left.2 \mathrm{H}, J=8 \mathrm{~Hz}, \underline{\mathrm{H}}_{2} \mathrm{C}-\mathrm{Th}-\mathrm{CHO}\right) ; 2.79(\mathrm{t}, 2 \mathrm{H}, J=8 \mathrm{~Hz}$, $\left.\underline{\mathrm{H}}_{2} \mathrm{C}-\mathrm{Th}-\mathrm{CH}=\mathrm{CH}\right) ; 1.68\left(\mathrm{~m}, 4 \mathrm{H}, \underline{\mathrm{H}}_{2} \mathrm{C}-\mathrm{CH}_{2}-\mathrm{Th}\right)$; $1.32\left(\mathrm{~m}, 16 \mathrm{H}, \mathrm{Th}-\left(\mathrm{CH}_{2}\right)_{2}-\left(\mathrm{CH}_{2}\right)_{4}\right)$.

MS $(\mathrm{m} / z): 592(100)\left(\mathbf{M}^{+}\right) ; 296(6)\left(\mathbf{M}^{2+}\right) ; 564(41)$ $(\mathrm{M}-\mathrm{CO})^{+} ; 327(26)\left(\mathrm{C}_{23} \mathrm{H}_{19} \mathrm{~S}\right)^{+}$.

\section{E,E-1-(5-Butyl-2-thienylvinyl)-4-(styryl)benzene (9)}

From $0.169 \mathrm{~g}(1 \mathrm{mmol})$ 5-butylthiophene-2-carbaldehyde, $0.535 \mathrm{~g}$ ( $1 \mathrm{mmol})$ triphenyl-(4-styrylbenzyl)phosphonium bromide [13] in $30 \mathrm{ml}$ absol. xylene, $1.400 \mathrm{~g}(10 \mathrm{mmol}) \mathrm{K}_{2} \mathrm{CO}_{3}$ and $0.0035 \mathrm{~g}$ (0.01 mmol) dibenzo[18]crown-6,18 h reflux; yellow crystals from EtOH; m.p. $123^{\circ} \mathrm{C}$; yield $0.278 \mathrm{~g}$ $(81 \%)$.

$$
\begin{array}{ccc}
\mathrm{C}_{24} \mathrm{H}_{24} \mathrm{~S}(344.5) & \\
\text { Calcd } & \mathrm{C} 83.67 & \text { H 7.02\%, } \\
\text { Found } & \text { C } 83.66 & \text { H 7.01\%. }
\end{array}
$$




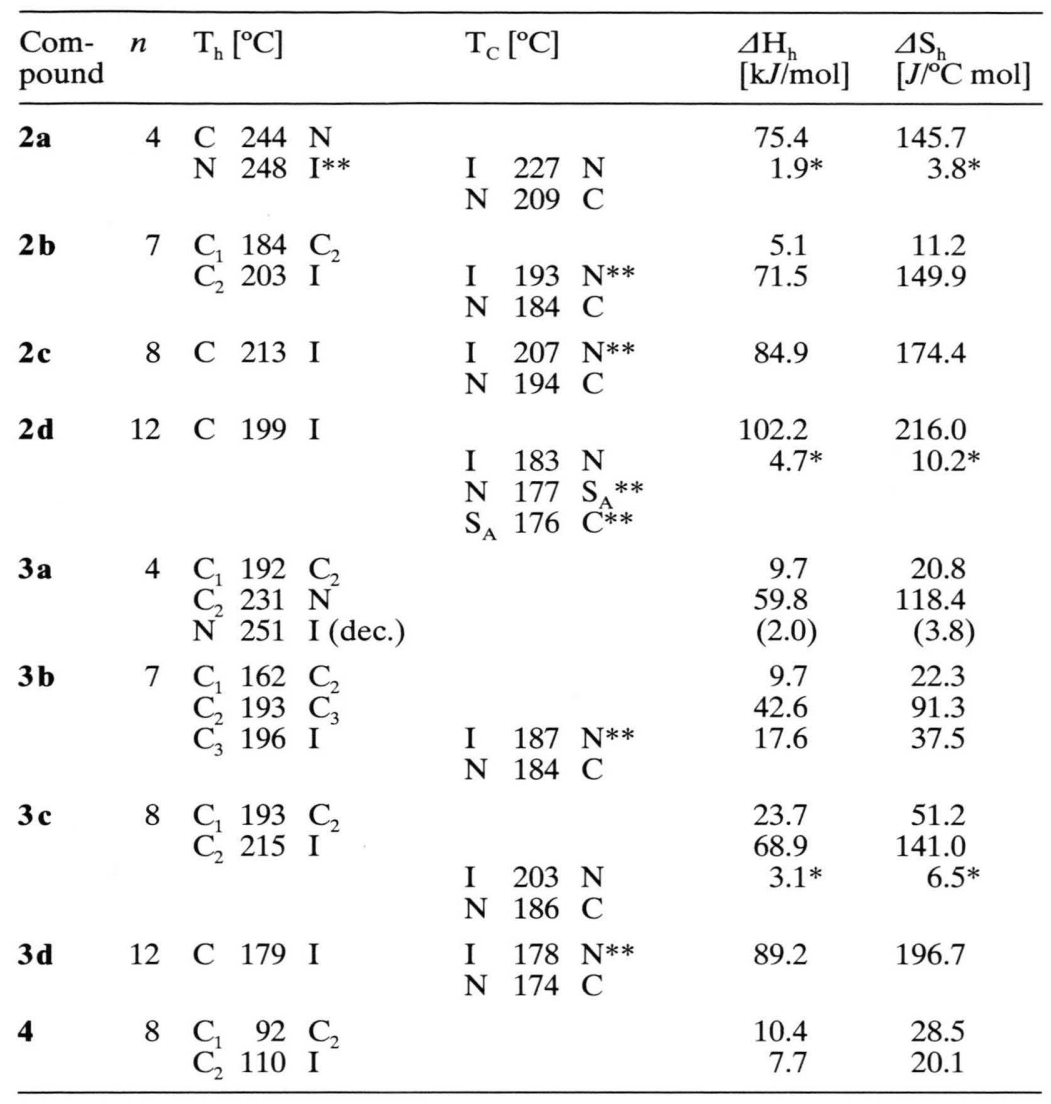

Table II. Thermal transitions of the E,E- $\alpha . \omega$-bis[5-(4-phenylstyryl)-2thienyl]alkanes $(\mathbf{2} \mathbf{a}-\mathbf{2} \mathbf{d})$, the E,E$\alpha . \omega$-bis [5-(4-phenylazophenylvinyl)-2-thienyl]alkanes $(\mathbf{3} \mathbf{a}-\mathbf{3} \mathbf{d})$ and E,E-1,8-bis\{2-[5-(1-anthracenyl)vinyl]thienyl octane (4) with different chain lengths $n ; \mathrm{T}_{\mathrm{h}}=$ transition temperature while heating, $\mathrm{T}_{\mathrm{C}}=$ transition temperature while cooling, $\Delta \mathrm{H}_{\mathrm{h}}=$ transition enthalpy while heating $(*=$ while cooling), $\Delta \mathrm{S}_{\mathrm{h}}=$ transition entropy while heating (* = while cooling), $\mathrm{C}=$ crystal, $\mathrm{N}=$ nematic, $\mathrm{I}=$ isotropic, dec. = decomposition, $* *=$ only veryfied by microscopy.

IR $(\mathrm{KBr}), \quad v\left(\mathrm{~cm}^{-1}\right)=3080(\mathrm{~m}), 3058(\mathrm{~m})$ $v(\mathrm{Ar}-\mathrm{C}-\mathrm{H}) ; 3025$ (s) $v\left(\mathrm{C}=\mathrm{C}_{\text {trans }}-\mathrm{H}\right) ; 2958(\mathrm{~s})$, 2925 (vs), 2862 (s) $v(\mathrm{C}-\mathrm{H}) ; 1622(\mathrm{~m}) v\left(\mathrm{C}=\mathrm{C}_{\text {conj. }}\right.$ ); $1538(\mathrm{w}) v(\mathrm{Ar}-\mathrm{C}=\mathrm{C}) ; 1468(\mathrm{~m}), 1449(\mathrm{~s}), 1418(\mathrm{~m})$ $v(\mathrm{Ar}) ; 955$ (vs) $\delta\left(\mathrm{C}=\mathrm{C}_{\text {trans }}-\mathrm{H}\right)_{\text {oop }} ; 812$ (vs) $\delta(\mathrm{Th}-\mathrm{C}-\mathrm{H})_{\text {oop }}$.

${ }^{1} \mathrm{H}$ NMR $\left(\mathrm{CDCl}_{3}\right) \delta(\mathrm{ppm})=7.0-7.6(\mathrm{~m}, 12 \mathrm{H}$, $\left.\mathrm{BH}_{8}, \mathrm{Vin}_{3}\right) ; 6.86\left(\mathrm{~d}, 1 \mathrm{H}, J_{3-4}=3.2 \mathrm{~Hz}, \mathrm{Th}-\mathrm{H}_{3}\right) ; 6.76$ $\left(\mathrm{d}, 1 \mathrm{H}, J_{2-1}=16.3 \mathrm{~Hz}, \mathrm{VinH}_{2}\right) ; 6.6\left(\mathrm{~d}, 1 \mathrm{H}, J_{4-3}=\right.$ $\left.3.2 \mathrm{~Hz}, \mathrm{Th}-\mathrm{H}_{4}\right) ; 2.8\left(\mathrm{t}, 2 \mathrm{H}, \mathrm{J}=8 \mathrm{~Hz}, \underline{\mathrm{H}}_{2} \mathrm{C}-\mathrm{Th}\right) ; 1.68$ (quintett, $2 \mathrm{H}, \mathrm{J}=5 \mathrm{~Hz}, \mathrm{H}_{2} \mathrm{C}-\mathrm{CH}_{2} \mathrm{Th}$ ); 1.38 (hex, $\left.2 \mathrm{H}, J=5 \mathrm{~Hz}, \underline{\mathrm{H}}_{2} \mathrm{C}-\left(\mathrm{CH}_{2}\right)_{2} \mathrm{Th}\right) ; 0.95(\mathrm{t}, 3 \mathrm{H}$, $\left.J=5 \mathrm{~Hz},-\mathrm{C}_{3}\right)$.

MS $(m / z): 344(100)\left(\mathbf{M}^{+}\right) ; 172(9)\left(M^{2+}\right) ; 301(47)$ $\left(\mathrm{C}_{21} \mathrm{H}_{17} \mathrm{~S}\right)^{+} ; 267(7)\left(\mathrm{C}_{21} \mathrm{H}_{15}\right)^{+}$.

\section{Results}

The thermal behaviour of the compounds is shown in Table II-IV. No mesophase was observed for the compounds of series 4. The compounds of the series 2 and 3 with 4 methylene groups already show enantiotropic, with 7, 8 and 12 meth- ylene groups monotropic nematic and respectively for $\mathbf{2 d}$ with 12 methylene groups also a small monotropic smectic phase which can be seen by microscopy (see Table II). In contrast to the low molecular weight compound 9 with no mesophases the compounds of series 5 exhibit while cooling the sample mostly several mesophases. Only 5 with 4 methylene groups melts exclusively enantiotropic into the nematic phase. With increasing chain length for the series $\mathbf{5}$ more and more mesophases have to be observed (see Fig. 1). So we see for $\mathbf{5 d}$, $5 \mathbf{e}$ and 5f with 9,10 and 12 methylene groups three mesophases respectively (see Fig. 2). Especially for 5d the temperature ranges are small for the smectic $\mathrm{A}$ and smectic 1 phase while heating the sample. So we could not observe a transition peak in the DSC for each transformation while heating the sample. We have assumed that each transformation peak has nearly a gauss shape so one part of the real DSCcurve can be described as a sum of three gauss curves (see Fig. 3). The compounds $\mathbf{7 a}$ and $\mathbf{7 c}$ with 4 and 8 methylene groups show exclusively enantiotropic 


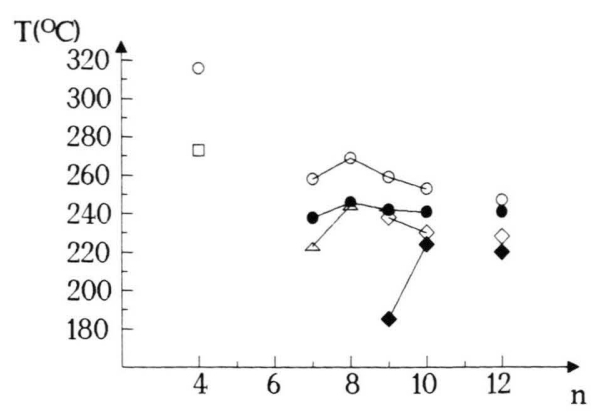

Fig. 1. Phase diagram of the all-E- $\alpha . \omega$-bis[5-(4-styrylstryryl)-2-thienyl]alkanes $(\mathbf{5} \mathbf{a}-\mathbf{5} \mathbf{f})$ with different chain lengths n; while cooling the sample $\bigcirc=$ transition temperature (t.t.) isotropic $\rightarrow$ nematic, $=$ t. t. nematic $\rightarrow$ smectic $\mathrm{A}, \diamond=$ t.t. smectic $\mathrm{A} \rightarrow$ smectic $1, \Delta$ t. t. smectic $\mathrm{A} \rightarrow$ solid, $>$ t. t. smectic $1 \rightarrow$ solid, $\square=$ nematic $\rightarrow$ solid.

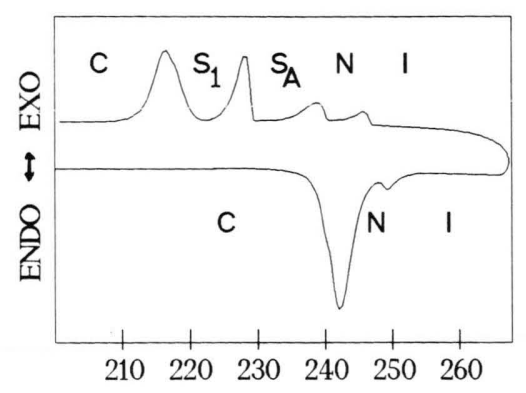

Fig. 2. DSC curve of $\mathbf{5 f}$ (with 12 methylene groups).

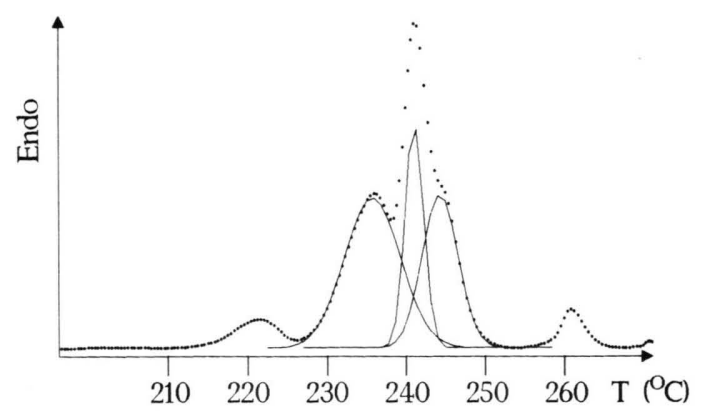

Fig. 3. Gauss fit on the endothermic DSC curve of $\mathbf{5} \mathbf{g}$ (with 9 methylene groups).

nematic and $\mathbf{7 b}$ and $\mathbf{7 d}$ with 7 and 12 methylene groups enantiotropic nematic and smectic A phases. For $\mathbf{7 b}$ we observed additionally a small smectic 1 phase over a small temperature range by microsco- py (see Table IV). The determination of the mesophases of the series $\mathbf{7}$ is complicated because the compounds begin to decompose in the nematic or isotropic phase also under a nitrogen atmosphere (see Table IV). The monoreacted products of the series $\mathbf{6}$ and $\mathbf{8}$ melt mostly directly into the isotropic phase. The only exceptions are the homologous compounds $8 \mathbf{a}$ and $8 \mathbf{c}$ with 4 and 8 methylene groups which are enantiotropic nematic (see Table IV).

All compounds are not thermally stable for longer heating times.

\section{Discussion}

Compounds with two mesogenic systems are quite of interest as model compounds for semi-flexible liquid crystalline polymers and also because their real properties differ from conventional low molecular weight compounds having a rigid core attached with one or more terminal alkyl chains or other groups. So, for example, the dimerization of one core can enhance or induce the formation of mesophases as shown for the compounds of the series 5 and 9. Corresponding to the polymers also in compounds with two mesogenic systems the formation of the mesophases is dependent on the inner chain length. Besides the known "even-odd effect" [12] (see below) the ratio of the spacer to core length is sometimes significant for building up special mesophases. In Fig. 1 the dependence of the transition temperatures on the length of the spacer is shown. Here and in Table III we can see that $\mathbf{5 d}$ with 9 methylene groups has an extended smectic mesophase while heating and cooling the sample. Supposing that the inner chain has the all-trans conformation and the $\mathrm{C}-\mathrm{C}$-bond the same repeating distance as determined on polyethylene crystals (2.5 $\AA$ ) [13] the length of the spacer with 8 or 9 methylene groups should be nearly $18.5-21 \AA$. Moreover one may estimate the length of the rigid core in series $\mathbf{5}$ with the s-trans-conformation of the thiophene system as about $17 \AA$ [17]. So the mesogenic groups in the compound can pack with higher efficiency in smectic structures if the chain length is similar or longer than the core length. Nevertheless the odd members of the series $\mathbf{5}$ and $\mathbf{7}$ especially with 7 and 9 methylene groups show a tendency to form smectic mesophases.

In Table III also the entropy changes $\Delta \mathrm{S}_{\mathrm{S}_{A} \rightarrow \mathrm{N}}$ at the smectic A-nematic transition of the series $\mathbf{5}$ are 
Table III. Thermal transitions of the E,E- $\alpha$. $\omega$-bis[5-(4-styrylstyryl)-2-thienyl]alkanes $(\mathbf{5} \mathbf{a}-\mathbf{5} \mathbf{f})$, with different chain lengths $n$ and E,E-1-(5-butyl-2-thienylvinyl)-4-(styryl)benzene (9); $\mathrm{T}_{\mathrm{h}}=$ transition temperature while heating, $\mathrm{T}_{\mathrm{C}}=$ transition temperature while cooling, $\Delta \mathrm{H}_{\mathrm{h}}=$ transition enthalpy while heating $(*=$ while cooling $), \Delta \mathrm{S}_{\mathrm{h}}=$ transition entropy while heating ( $*$ while cooling), $\mathrm{C}=$ crystal, $\mathrm{S}=$ smectic, $\mathrm{N}=$ nematic, $\mathrm{I}=$ isotropic, dec. $=$ decomposition, $* *=$ only veryfied by microscopy, $* * *=$ veryfied by gauss-fit of the DSC-curve.

\begin{tabular}{|c|c|c|c|c|c|c|c|c|c|c|}
\hline \multirow{2}{*}{$\begin{array}{l}\text { Com- } \\
\text { pound }\end{array}$} & \multirow{2}{*}{$\begin{array}{l}n \\
4\end{array}$} & \multicolumn{3}{|c|}{$\mathrm{T}_{\mathrm{h}}\left[{ }^{\circ} \mathrm{C}\right]$} & \multicolumn{3}{|c|}{$\mathrm{T}_{\mathrm{C}}\left[{ }^{\circ} \mathrm{C}\right]$} & \multirow{2}{*}{$\frac{\mathrm{T}_{\mathrm{C}}\left(\mathrm{N} \rightarrow \mathrm{S}_{\mathrm{A}}\right)}{\mathrm{T}_{\mathrm{C}}(\mathrm{I} \rightarrow \mathrm{N})}$} & \multirow{2}{*}{$\left.\begin{array}{l}\Delta \mathrm{H}_{\mathrm{h}} \\
{[\mathrm{k} J / \mathrm{mol}]}\end{array}\right]$} & \multirow{2}{*}{$\begin{array}{c}\Delta \mathrm{S}_{\mathrm{h}} \\
{\left[\mathrm{J} /{ }^{\circ} \mathrm{C} \text { mol }\right]}\end{array}$} \\
\hline & & $\begin{array}{l}\mathrm{C} \\
\mathrm{N}\end{array}$ & $\begin{array}{l}288 \\
316\end{array}$ & $\begin{array}{l}\mathrm{N} \\
\mathrm{I} \text { (dec.) }\end{array}$ & $\mathrm{N}$ & 273 & C & & & \\
\hline $5 b$ & 7 & $\begin{array}{l}\mathrm{C} \\
\mathrm{N}\end{array}$ & $\begin{array}{l}246 \\
262\end{array}$ & $\begin{array}{l}\mathrm{N} \\
\mathrm{I}\end{array}$ & $\begin{array}{l}\mathrm{I} \\
\mathrm{N} \\
\mathrm{S}_{\mathrm{A}}\end{array}$ & $\begin{array}{l}258 \\
238 \\
223\end{array}$ & $\begin{array}{l}\mathrm{N} \\
\mathrm{S}_{\mathrm{A}} \\
\mathrm{C}\end{array}$ & 0.922 & $\begin{array}{c}62.8 \\
2.8 \\
28.0^{*}\end{array}$ & $\begin{array}{c}120.9 \\
5.2 \\
54.7^{*}\end{array}$ \\
\hline $5 c$ & 8 & $\begin{array}{l}\mathrm{C}_{1} \\
\mathrm{C}_{2} \\
\mathrm{~N}^{-}\end{array}$ & $\begin{array}{l}221 \\
256 \\
273\end{array}$ & $\begin{array}{l}\mathrm{C}_{2} \\
\mathrm{~N} \\
\mathrm{I}\end{array}$ & $\begin{array}{l}\mathrm{I} \\
\mathrm{N} \\
\mathrm{S}_{\mathrm{A}}\end{array}$ & $\begin{array}{l}269 \\
246 \\
244\end{array}$ & $\begin{array}{l}\mathrm{N} \\
\mathrm{S}_{\mathrm{A}}{ }^{* *}\end{array}$ & 0.914 & $\begin{array}{r}10.5 \\
78.2 \\
6.8\end{array}$ & $\begin{array}{r}21.2 \\
147.5 \\
12.4\end{array}$ \\
\hline $5 \mathrm{~d}$ & 9 & $\begin{array}{l}\mathrm{C}_{1} \\
\mathrm{C}_{2} \\
\mathrm{~S}_{1} \\
\mathrm{~S}_{\mathrm{A}} \\
\mathrm{N}\end{array}$ & $\begin{array}{l}214 \\
227 \\
238 \\
242 \\
259\end{array}$ & $\begin{array}{l}\mathrm{C}_{2} \\
\mathrm{~S}_{1} \\
\mathrm{~S}_{\mathrm{A}} \\
\mathrm{N} \\
\mathrm{I}\end{array}$ & $\begin{array}{l}\mathrm{I} \\
\mathrm{N} \\
\mathrm{S}_{\mathrm{A}} \\
\mathrm{S}_{1}\end{array}$ & $\begin{array}{l}259 \\
242 \\
238 \\
185\end{array}$ & $\begin{array}{l}\mathrm{N} \\
\mathrm{S}_{\mathrm{A}} \\
\mathrm{S}_{1} \\
\mathrm{C}\end{array}$ & 0.934 & $\begin{array}{l}3.9 \\
24.9^{* * *} \\
12.2^{* * *} \\
16.1^{* * *} \\
2.6\end{array}$ & $\begin{array}{c}8.0 \\
49.7^{* * *} \\
23.8^{* * *} \\
31.3^{* * *} \\
4.8\end{array}$ \\
\hline $5 e$ & 10 & $\begin{array}{l}\mathrm{C}_{1} \\
\mathrm{C}_{2} \\
\mathrm{~S}_{\mathrm{A}} \\
\mathrm{N}\end{array}$ & $\begin{array}{l}210 \\
246 \\
248 \\
257\end{array}$ & $\begin{array}{l}\mathrm{C}_{2} \\
\mathrm{~S}_{\mathrm{A}} \\
\mathrm{N}^{* *} \\
\mathrm{I}\end{array}$ & $\begin{array}{l}\mathrm{I} \\
\mathrm{N} \\
\mathrm{S}_{\mathrm{A}} \\
\mathrm{S}_{1}\end{array}$ & $\begin{array}{l}253 \\
241 \\
230 \\
224\end{array}$ & $\begin{array}{l}\mathrm{N} \\
\mathrm{S}_{\mathrm{A}}^{* *} \\
\mathrm{~S}_{1} * * \\
\mathrm{C}^{* *}\end{array}$ & 0.952 & $\begin{array}{r}12.5 \\
78.2 \\
7.1\end{array}$ & $\begin{array}{r}25.8 \\
150.6 \\
\\
13.4\end{array}$ \\
\hline $5 \mathrm{f}$ & 12 & $\begin{array}{l}\mathrm{C} \\
\mathrm{S}_{1} \\
\mathrm{~S}_{\mathrm{A}} \\
\mathrm{N}\end{array}$ & $\begin{array}{l}238 \\
240 \\
242 \\
247\end{array}$ & $\begin{array}{l}\mathrm{S}_{1} \\
\mathrm{~S}_{\mathrm{A}} * * \\
\mathrm{~N}^{* *} \\
\mathrm{I}\end{array}$ & $\begin{array}{l}\mathrm{I} \\
\mathrm{N} \\
\mathrm{S}_{\mathrm{A}} \\
\mathrm{S}_{1}\end{array}$ & $\begin{array}{l}247 \\
241 \\
228 \\
220\end{array}$ & $\begin{array}{l}\mathrm{N} \\
\mathrm{S}_{\mathrm{A}} \\
\mathrm{S}_{1} \\
\mathrm{C}\end{array}$ & 0.976 & $\begin{array}{c}5.4 \\
8.4^{*} \\
18.4^{*} \\
36.1^{*}\end{array}$ & $\begin{array}{l}10.4 \\
16.4^{*} \\
36.7^{*} \\
73.1^{*}\end{array}$ \\
\hline 9 & & $\mathrm{C}$ & 208 & I & I & 207 & $\mathrm{C}$ & & 22.3 & 46.2 \\
\hline
\end{tabular}

listed. It is seen easily that with increasing chain length the $\Delta \mathrm{S}_{\mathrm{S}_{\mathrm{A}} \rightarrow \mathrm{N}}$-values are decreased. So for $\mathbf{5} \mathbf{b}$ with 7 methylene groups $\Delta \mathrm{S}_{\mathrm{S}_{\mathrm{A}} \rightarrow \mathrm{N}}$ is $54.7 \mathrm{~J} /{ }^{\circ} \mathrm{C} \mathrm{mol}$ whereas for $\mathbf{5 f}$ with 12 methylene groups it is only $16.4 \mathrm{~J} /{ }^{\circ} \mathrm{C}$ mol. This tendency was observed earlier by McMillian [14] and confirmed by Hogan et al. [15] for different compounds. According to that this tendency of the entropy values are not referred to different molecular structures but to the broadness of the temperature range of the nematic phase of the compound. So the $\Delta \mathrm{S}_{\mathrm{S}_{\mathrm{A}} \rightarrow \mathrm{N}}$-values are predicted to decrease as the ratio $\mathrm{T}_{\mathrm{S}_{\mathrm{A}} \rightarrow \mathrm{N}} / \mathrm{T}_{\mathrm{N} \rightarrow \mathrm{I}}$ of the transition temperatures deviates from unity. As seen in Table III these temperature ratios are decreasing in error with decreasing chain length. So we assume that the $\Delta \mathrm{S}_{\mathrm{S}_{\mathrm{A}} \rightarrow \mathrm{N}}$-values for the homologous compounds $5 \mathbf{c}$ and $\mathbf{5 g}$ with 8 and 10 methylene groups which we can not determine by DSC are in the line of the values for the compounds $5 \mathbf{b}, 5 \mathbf{d}$ and $5 \mathbf{f}$ (with 7, 9 and 12 methylene groups). Moreover the $\Delta \mathrm{S}_{\mathrm{S}_{\mathrm{A}} \rightarrow \mathrm{N}}$-values (determinated by the described gauss-fit) for the homologous compound 5 e with 9 methylene groups are verified by the known trend 


\begin{tabular}{|c|c|c|c|c|c|c|c|c|c|}
\hline \multirow{2}{*}{$\begin{array}{l}\text { Com- } \\
\text { pound } \\
6 \mathbf{a}\end{array}$} & \multirow{2}{*}{$\begin{array}{l}n \\
4\end{array}$} & \multicolumn{3}{|c|}{$\mathrm{T}_{\mathrm{h}}\left[{ }^{\circ} \mathrm{C}\right]$} & \multicolumn{3}{|c|}{$\mathrm{T}_{\mathrm{C}}\left[{ }^{\circ} \mathrm{C}\right]$} & \multirow{2}{*}{$\begin{array}{l}\Delta \mathrm{H}_{\mathrm{h}} \\
{[\mathrm{k} J / \mathrm{mol}]}\end{array}$} & \multirow{2}{*}{$\begin{array}{l}\begin{array}{l}\Delta \mathrm{S}_{\mathrm{h}} \\
{\left[\mathrm{J} /{ }^{\circ} \mathrm{C} \mathrm{mol}\right]}\end{array} \\
53.4 \\
39.2\end{array}$} \\
\hline & & $\begin{array}{l}\mathrm{C}_{1} \\
\mathrm{C}_{2}\end{array}$ & $\begin{array}{l}155 \\
185\end{array}$ & $\begin{array}{l}\mathrm{C}_{2} \\
\mathrm{I}\end{array}$ & I & 185 & $\mathrm{C}$ & & \\
\hline $6 b$ & 12 & $\begin{array}{l}\mathrm{C}_{1} \\
\mathrm{C}_{2} \\
\mathrm{C}_{3}\end{array}$ & $\begin{array}{l}120 \\
126 \\
157\end{array}$ & $\begin{array}{l}\mathrm{C}_{2} \\
\mathrm{C}_{3} \\
\mathrm{I}\end{array}$ & $\begin{array}{l}\mathrm{I} \\
\mathrm{C}_{1},\end{array}$ & $\begin{array}{l}156 \\
105\end{array}$ & $\begin{array}{l}\mathrm{C}_{1^{\prime}} \\
\mathrm{C}_{2^{\prime}}\end{array}$ & $\begin{array}{r}9.8 \\
20.1 \\
14.1\end{array}$ & $\begin{array}{l}24.9 \\
50.2 \\
32.8\end{array}$ \\
\hline $7 \mathbf{a}$ & 4 & $\begin{array}{l}\mathrm{C}_{1} \\
\mathrm{C}_{2} \\
\mathrm{~N}^{2}\end{array}$ & $\begin{array}{l}244 \\
286 \\
293\end{array}$ & $\begin{array}{l}\mathrm{C}_{2} \\
\mathrm{~N} \\
\mathrm{I} \text { (dec.) }\end{array}$ & & & & $\begin{array}{c}6.8 \\
51.9 \\
(1.9)\end{array}$ & $\begin{array}{l}13.2 \\
92.6 \\
(3.4)\end{array}$ \\
\hline $7 b$ & 7 & $\begin{array}{l}\mathrm{C} \\
\mathrm{S}_{\mathrm{A}} \\
\mathrm{N}\end{array}$ & $\begin{array}{l}253 \\
258 \\
273\end{array}$ & $\begin{array}{l}\mathrm{S}_{\mathrm{A}} \\
\mathrm{N}^{* *} \\
\mathrm{I} \text { (dec.) }\end{array}$ & $\begin{array}{l}\mathrm{N} \\
\mathrm{S}_{\mathrm{A}} \\
\mathrm{S}_{1}\end{array}$ & $\begin{array}{l}244 \\
235 \\
232\end{array}$ & $\begin{array}{l}\mathrm{S}_{\mathrm{A}} * * \\
\mathrm{~S}_{1} * * \\
\mathrm{C}^{* *}\end{array}$ & $\begin{array}{l}70.8 \\
(1.9)\end{array}$ & $\begin{array}{r}134.4 \\
(3.5)\end{array}$ \\
\hline $7 c$ & 8 & $\begin{array}{l}\mathrm{C}_{1} \\
\mathrm{C}_{2} \\
\mathrm{C}_{3} \\
\mathrm{~N}^{2}\end{array}$ & $\begin{array}{l}230 \\
237 \\
259 \\
275\end{array}$ & $\begin{array}{l}\mathrm{C}_{2} \\
\mathrm{C}_{3} \\
\mathrm{~N}^{-} \\
\mathrm{I}(\text { dec. })\end{array}$ & $\mathrm{N}$ & 245 & $\mathrm{C}$ & $\begin{array}{r}8.3 \\
0.7 \\
85.8\end{array}$ & $\begin{array}{r}16.5 \\
1.3 \\
160.9\end{array}$ \\
\hline $7 d$ & 12 & $\begin{array}{l}\mathrm{C}_{1} \\
\mathrm{C}_{2} \\
\mathrm{~S}_{\mathrm{A}} \\
\mathrm{N}\end{array}$ & $\begin{array}{l}231 \\
238 \\
249 \\
257\end{array}$ & $\begin{array}{l}\mathrm{C}_{2} \\
\mathrm{~S}_{\mathrm{A}} \\
\mathrm{N} \\
\mathrm{I}(\text { dec. })\end{array}$ & $\begin{array}{l}\mathrm{N} \\
\mathrm{S}_{\mathrm{A}}\end{array}$ & $\begin{array}{l}237 \\
212\end{array}$ & $\begin{array}{l}\mathrm{S}_{\mathrm{A}} \\
\mathrm{C}\end{array}$ & $\begin{array}{r}9.8 \\
98.3 \\
10.1\end{array}$ & $\begin{array}{r}19.4 \\
192.4 \\
19.3\end{array}$ \\
\hline $8 \mathbf{a}$ & 4 & $\begin{array}{l}\mathrm{C}_{1} \\
\mathrm{C}_{2} \\
\mathrm{~N}^{2}\end{array}$ & $\begin{array}{l}172 \\
197 \\
213\end{array}$ & $\begin{array}{l}\mathrm{C}_{2} \\
\mathrm{~N} \\
\mathrm{I}\end{array}$ & $\begin{array}{l}\mathrm{I} \\
\mathrm{N}\end{array}$ & $\begin{array}{l}210 \\
193\end{array}$ & $\begin{array}{l}\mathrm{N} \\
\mathrm{C}\end{array}$ & $\begin{array}{r}23.1 \\
11.7 \\
0.5\end{array}$ & $\begin{array}{r}51.9 \\
24.9 \\
1.0\end{array}$ \\
\hline $8 \mathbf{b}$ & 7 & $\mathrm{C}$ & 180 & I & I & 180 & $\mathrm{C}$ & 13.5 & 29.9 \\
\hline $8 c$ & 8 & $\begin{array}{l}\mathrm{C} \\
\mathrm{N}\end{array}$ & $\begin{array}{l}181 \\
189\end{array}$ & $\begin{array}{l}\mathrm{N} \\
\mathrm{I}\end{array}$ & $\begin{array}{l}\mathrm{I} \\
\mathrm{N}\end{array}$ & $\begin{array}{l}189 \\
179\end{array}$ & $\begin{array}{l}\mathrm{N} \\
\mathrm{C}\end{array}$ & $\begin{array}{r}16.7 \\
1.2\end{array}$ & $\begin{array}{r}36.7 \\
2.5\end{array}$ \\
\hline $8 d$ & 12 & $\mathrm{C}$ & 169 & I & I & 169 & $\mathrm{C}$ & 16.0 & 36.1 \\
\hline
\end{tabular}

Table IV. Thermal transitions of the E,E-1-(5-formyl-2-thienyl)$\omega$-[5-(4-(styryl)styryl)-2-thienyl]alkanes $(\mathbf{6} \mathbf{a}, \mathbf{6 b})$, all-E,E- $\alpha . \omega$-bis\{5-[4-(4-phenyl-buta-1.3-dienyl)styryl]-2-thienyl alkanes $(\mathbf{7} \mathbf{a}-\mathbf{7} \mathbf{d})$, all-E-1-(5-formyl-2-thienyl)- $\omega$ \{5-[4-(4-phenyl-1.3-dienyl)styryl]2-thienyl $\}$ alkanes $(\mathbf{8} \mathbf{a}-\mathbf{8 d})$ with different chain length $n ; \mathrm{T}_{\mathrm{h}}=$ transition temperature while heating, $\mathrm{T}_{\mathrm{C}}=$ transition temperature while cooling, $\Delta \mathrm{H}_{\mathrm{h}}=$ transition enthalpy while heating, $\Delta \mathrm{S}_{\mathrm{h}}=$ transition entropy while heating, $\mathrm{C}=$ crystal, $\mathrm{S}=$ smectic, $\mathrm{N}=$ nematic, $\mathrm{I}=$ isotropic, dec. $=$ decomposition, $* *=$ only veryfied by microscopy.

(see Fig. 3).

In contrast to the entropies $\Delta \mathrm{S}_{\mathrm{S}_{\mathrm{A}} \rightarrow \mathrm{N}}$ the transition entropies from the nematic to the isotropic phase $\Delta \mathrm{S}_{\mathrm{N} \rightarrow \mathrm{I}}$ of the series 5 show a quite different behaviour. So these entropies alternate with increasing chain length; compounds with a spacer containing an even number of methylene groups have the higher values than those one with odd numbers of methylene groups (see Table III). This "even-odd effect" for these compounds is well-known in literature [12] and is often related to different orientation of the moieties in the nematic phases by different chain length in such a compound with two mesogenic groups [16]. A comparison to the series 2, 3 and $\mathbf{7}$ is not possible because respectively for the series $\mathbf{2}$ and $\mathbf{3}$ some transformations from the isotropic to the nematic phase could only observed by microscopy and for the series 7 the clearing points of all homologous compounds were combined by a decomposition of each sample.

Except for the series $\mathbf{6}$ in all compounds the thiophene and benzene systems are bound in the same sequences in the core (see Scheme 5). Their differences are only the result of different connecting

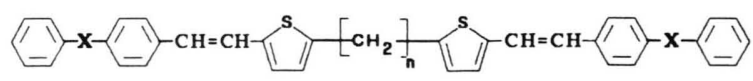

Scheme 5. Schematic formula of the compounds of the series $\mathbf{2}(\mathbf{X}=-), \mathbf{3}(\mathbf{X}=-\mathrm{N}=\mathrm{N}), \mathbf{5}(\mathbf{X}=-\mathrm{CH}=\mathrm{CH})$ and $\mathbf{7}(\mathbf{X}=-\mathrm{CH}=\mathrm{CH}-\mathrm{CH}=\mathrm{CH}-)$. 


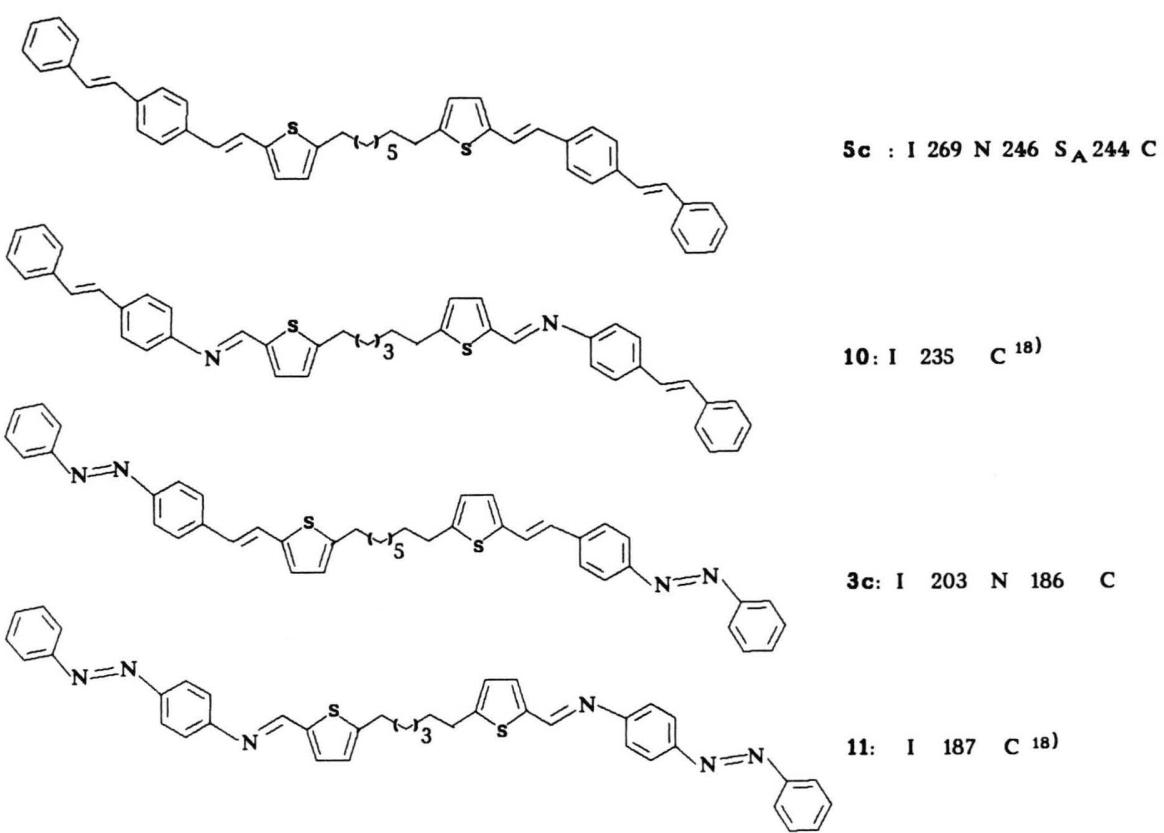

Scheme 6. Liquid crystalline properties of some vinylenes and azomethines with analogous structures. groups between the ring systems like vinylene, azo or 1.4-buta-1.3-dienyl groups. So the comparison between the compounds shows that only the compounds with vinylene groups melt with extended mesophases. This fact can not explained exclusively with the structure behaviour of the compounds. Although 2a-2d have no azo groups in contrast to the series 3 they melt similar to the "dimeric" compounds of the series 3 with an azo group and series 4 with a vinylene group in the core. Moreover the compounds of the series 7 with the greatest number of vinylene groups in each core show while heating the sample mesophases over an extended temperature range. A further example is given by a comparison to the azomethine derivatives E,E-1.6-bis\{5-[4-(styrylphenyl)iminomethyl]-2-thienyl\}hexane (10) [18] and E,E-1.6-bis $\{5-[\mathrm{N}-(4-$ phenylazophenyl)iminomethyl]-2-thienyl hexane (11) [18] which are in contrast to the vinylenes of the series $\mathbf{3}$ and $\mathbf{5}$ not liquid crystalline (see Scheme 6). Similar results were observed by Koßmehl and Budwill [19] on low molecular weight compounds assuming that the difference of the mesophase behaviour caused by different structural arrangements in the vinylene and azomethine group. Moreover we additionally assume that the deviation from the electronic structure should have an effect on the different thermal behaviour of the 
[1] G. Koßmehl, F. D. Hoppe, B. Hirsch, Z. Naturforsch. $48 \mathrm{~b}, 826$ (1993).

[2] K. Nakasuji, S. Akiyama, M. Nakagawa, Bull. Chem. Soc. Jpn. 45 (3), 890 (1972).

[3] G. Drehfahl, G. Plötner, Chem. Ber. 94, 907 (1961).

[4] G. A. Lapitskii, S. M. Makin, A. A. Berlin, Vysokomol. Soedin. 9, 1274 (1967).

[5] G. C. A. Bellinger, W. E. Campbell, R. G. F. Giles, J. D. Tobias, J. Chem. Soc. Perkin Trans. I 1982, 2819.

[6] R. M. Boden, Synthesis 1975, 784.

[7] C. A. van der Werf, W. E. Mc Ewen, M. Zanger, J. Am. Chem. Soc. 81, 3806 (1959).

[8] G. Aksnes, J. Songstad, Acta Chem. Scand. 16, 1460 (1962).

[9] W. Allen, B. G. Hutley, J. Chem. Soc. Perkin Trans. 2, 67 (1972).

[10] M. Grayson, P. T. Keough, J. Am. Chem. Soc. 82, 3919 (1960).
[11] G. Koßmehl, J. Wallis, Makromol. Chem. 183, 331 (1982).

[12] A. C. Griffin, T. R. Britt, J. Am. Chem. Soc. 103, 4957 (1981)

[13] F. A. Bovey, T. K. Kwei, in F. A. Bovey, F. H. Winslow (eds): "Macromolecules", p. 259, Academic Press, New York, N. Y. (1979).

[14] W. L. Mc Millan, Phys. Rev. A 4, 1238 (1972).

[15] J. L. Hogan, C. T. Imrie, G. R. Luckhurst, Liq. Crystals 3 (5), 645 (1988).

[16] J. A. Buglione, A. Roviello, A. Sirigu, Mol. Cryst. Liq. Cryst. 106, 169 (1984).

[17] G. Ruban, D. Zobel, Acta Crystallogr. B 31, 2632 (1975).

[18] G. Koßmehl, J. Bahr, Z. Naturforsch. 46b, 245 (1991).

[19] G. Koßmehl, D. Budwill, Z. Naturforsch. 42 b, 478 (1987). 